
\title{
Research S Surare \\ Vehicle Lateral Motion State Estimation Based on Adaptive Cubature Kalman Filter
}

\section{Zhi-cheng HE}

Hunan University

\section{Zhen-yu ZHANG ( $\nabla 2523138212 @ q q . c o m$ )}

master at College of Mechanical and Vehicle Engineering, Hunan University, China

https://orcid.org/0000-0003-4292-5223

\section{En-lin ZHOU}

Hunan University

\section{Bao-Iv WEI}

Hunan University

\section{Original Article}

Keywords: State estimation, Vehicle system, Adaptive cubature Kalman filter, Adaptive proportion integral observer, Partition coefficient

Posted Date: December 15th, 2020

DOI: https://doi.org/10.21203/rs.3.rs-126110/v1

License: (c) (i) This work is licensed under a Creative Commons Attribution 4.0 International License.

Read Full License 


\section{Chinese Journal of Mechanical Engineering Vehicle Lateral Motion State Estimation Based on Adaptive Cubature Kalman Filter --Manuscript Draft--}

\begin{tabular}{|c|c|c|}
\hline Manuscript Number: & \multicolumn{2}{|l|}{ CHME-D-20-00737 } \\
\hline Full Title: & \multicolumn{2}{|c|}{ Vehicle Lateral Motion State Estimation Based on Adaptive Cubature Kalman Filter } \\
\hline Article Type: & \multicolumn{2}{|l|}{ Original Article } \\
\hline Section/Category: & \multicolumn{2}{|l|}{ Advanced Transportation Equipment } \\
\hline \multirow[t]{3}{*}{ Funding Information: } & $\begin{array}{l}\text { National Natural Science Foundation of } \\
\text { China } \\
\text { (U1864207) }\end{array}$ & Mr Zhi-cheng HE \\
\hline & $\begin{array}{l}\text { State Key Laboratory of Advanced } \\
\text { Technologies for Comprehensive } \\
\text { Utilization of Platinum Metals (CN) } \\
\text { (71975004) }\end{array}$ & Mr Zhi-cheng HE \\
\hline & $\begin{array}{l}\text { Science and Technology Bureau, } \\
\text { Changsha (CN) } \\
\text { (kq1907104) }\end{array}$ & Mr Zhi-cheng HE \\
\hline Abstract: & \multicolumn{2}{|c|}{$\begin{array}{l}\text { Abstract: Accurate and reliable vehicle state information is very significant to the } \\
\text { vehicle lateral stability control, while it is hard to get information such as body sideslip } \\
\text { angle and lateral tire forces due to lack the nonlinearity of the tire. This paper presents } \\
\text { a new combined method (adaptive cubature Kalman filter(ACKF) and adaptive } \\
\text { proportion integral observer(APIO)) to estimate body sideslip angle, yaw rate and } \\
\text { lateral tire force for vehicle system. Firstly, based on a four-wheel vehicle dynamics } \\
\text { model, (ACKF) is used to estimate body sideslip angle and yaw rate with considering } \\
\text { the nonlinear lateral tire force stage. Due to system nonlinearities and un-modeled } \\
\text { dynamics, APIO is used to improve the estimated body sideslip angle by utilizing the } \\
\text { estimated yaw rate and vehicle lateral speed. Then, ACKF is used to estimate the front } \\
\text { and rear lateral tire forces based on the one-order tire dynamics model. By utilizing the } \\
\text { partition coefficient calculated by the vertical force model, the front and rear lateral tire } \\
\text { forces are further distributed to left and right wheels. } \\
\text { For comparison, estimation model based on extended Kalman filter(EKF) is built and } \\
\text { investigated. Simulation using Matlab/Simulink-CarSim and car test verifies the } \\
\text { effectiveness of the proposed method. }\end{array}$} \\
\hline Corresponding Author: & \multicolumn{2}{|l|}{$\begin{array}{l}\text { Zhen-yu ZHANG, M.D. } \\
\text { Hunan University } \\
\text { Changsha, Hu nan CHINA }\end{array}$} \\
\hline Corresponding Author E-Mail: & \multicolumn{2}{|l|}{ 2523138212@qq.com } \\
\hline \multicolumn{3}{|l|}{$\begin{array}{l}\text { Corresponding Author Secondary } \\
\text { Information: }\end{array}$} \\
\hline Corresponding Author's Institution: & \multicolumn{2}{|l|}{ Hunan University } \\
\hline \multicolumn{3}{|l|}{$\begin{array}{l}\text { Corresponding Author's Secondary } \\
\text { Institution: }\end{array}$} \\
\hline First Author: & \multicolumn{2}{|l|}{ Zhi-cheng HE, Phd } \\
\hline \multicolumn{3}{|l|}{ First Author Secondary Information: } \\
\hline \multirow[t]{4}{*}{ Order of Authors: } & \multicolumn{2}{|l|}{ Zhi-cheng HE, Phd } \\
\hline & \multicolumn{2}{|l|}{ Zhen-yu ZHANG, M.D. } \\
\hline & \multicolumn{2}{|l|}{ En-lin ZHOU } \\
\hline & \multicolumn{2}{|l|}{ Bao-lv WEI } \\
\hline \multicolumn{3}{|c|}{ Order of Authors Secondary Information: } \\
\hline \multicolumn{3}{|l|}{ Suggested Reviewers: } \\
\hline Opposed Reviewers: & & \\
\hline
\end{tabular}


Additional Information:

Question

Response

Powered by Editorial Manager $\AA^{\circledR}$ and ProduXion Manager $\AA$ from Aries Systems Corporation 
1

2

3

4

5

6

7

\section{Title page}

\section{Vehicle Lateral Motion State Estimation Based on Adaptive Cubature Kalman Filter}

Zhi-Cheng He, born in 1983, is currently an associate professor at College of Mechanical and Vehicle Engineering, Hunan University, China. He received his PhD from Hunan University, China, in 2011. His research interests include man-machine system and intelligent robotics.

Tel: +0731-88821445; E-mail: hezhicheng815@gmail.com, hezhicheng815@163.com

Zhen-Yu Zhang, born in 1995, is currently a master at College of Mechanical and Vehicle Engineering, Hunan University, China. E-mail: 2523138212@qq.com, zhangzhenyuhuda@163.com

En-Lin Zhou, born in 1985, is currently a PhD at College of Mechanical and Vehicle Engineering, Hunan University, China.

E-mail:tenrey18@163.com

Bao-lv Wei, born in 1978, is currently a PhD at College of Mechanical and Vehicle Engineering, Hunan University, China.

E-mail: baolv.wei@sgmw.com.cn

Corresponding author: Zhen-Yu Zhang E-mail: 2523138212@qq.com 
ORIGINAL ARTICLE

\title{
Vehicle Lateral Motion State Estimation Based on Adaptive Cubature Kalman Filter
}

\author{
Zhi-Cheng He ${ }^{1,2} \cdot$ Zhen-Yu Zhang* 1,2 $\bullet$ Enlin Zhou ${ }^{1,2} \cdot B a o-L v ~ W e i{ }^{1,2}$
}

(c) Chinese Mechanical Engineering Society and Springer-Verlag Berlin Heidelberg 2020

\begin{abstract}
Accurate and reliable vehicle state information is very significant to the vehicle lateral stability control, while it is hard to get information such as body sideslip angle and lateral tire forces due to lack the nonlinearity of the tire. This paper presents a new combined method (adaptive cubature Kalman filter(ACKF) and adaptive proportion integral observer(APIO)) to estimate body sideslip angle, yaw rate and lateral tire force for vehicle system. Firstly, based on a four-wheel vehicle dynamics model, (ACKF) is used to estimate body sideslip angle and yaw rate with considering the nonlinear lateral tire force stage. Due to system nonlinearities and un-modeled dynamics, APIO is used to improve the estimated body sideslip angle by utilizing the estimated yaw rate and vehicle lateral speed. Then, ACKF is used to estimate the front and rear lateral tire forces based on the one-order tire dynamics model. By utilizing the partition coefficient calculated by the vertical force model, the front and rear lateral tire forces are further distributed to left and right wheels.

For comparison, estimation model based on extended Kalman filter(EKF) is built and investigated. Simulation using Matlab/Simulink-CarSim and car test verifies the effectiveness of the proposed method.
\end{abstract}

Keywords: State estimation • Vehicle system - Adaptive cubature Kalman filter $•$ Adaptive proportion integral observer $•$ Partition coefficient

\section{Introduction}

Zhen-Yu Zhang 2523138212@qq.com

1 College of Mechanical and Vehicle Engineering, Hunan University, Changsha 410082, China

2 State Key Laboratory of Advanced Design and Manufacturing for Vehicle Body, Hunan University, Changsha 410082, China
With the development of automotive technology, the lateral stability of vehicle has attracted more and more attention from researchers. For the research on the lateral stability of vehicle, it is necessary to use sensors to obtain real-time status information of vehicle, such as longitudinal speed, lateral speed, lateral acceleration, yaw rate, and body sideslip angle. However, due to expensive equipment, mass production models will not be equipped with sensors for body sideslip angle. Therefore the currently adopted measures are utilizing some estimation methods to get body sideslip angle [1-5].

Common vehicle state estimation methods include sliding mode observer (SMO), Luenberger observer, non-linear observers, and Kalman filters. Among them, Kalman filter and its improved algorithms have become a research focus because of their high stability and accuracy. Chen et al. [6] proposed an estimation method combining strong tracking filter with extended Kalman filter which overcomes the tire lateral force and longitudinal force calculation problems when the longitudinal acceleration and lateral acceleration are unknown. The test proved that the method can effectively estimate the tire longitudinal and lateral forces. Geng et al. [7] proposed a noise adaptive extended Kalman filter algorithm to estimate the state information including body sideslip angle and yaw rate in view of the problem that the estimation accuracy is reduced due to inaccurate prior information. Simulation showed that the method achieves effective estimation. Similarly, Zhang et al. [8] proposed an extended Kalman filter algorithm based on the adaptive strategy of sliding window of innovation sequence, which improved the estimation accuracy of body sideslip angle. Moreover, in [9], an augmented EKF observer considering model parameters variability and noisy measurement input 
was proposed for body sideslip angle and yaw rate estimation. Chen et al. [10] combined EKF with Luenberger observer and high-order sliding mode observer to estimate tire longitudinal force, lateral vehicle speed, body sideslip angle and yaw rate. Results showed an improvement in the estimation accuracy compared to standard EKF. Similarly, to minimize the effects of sensors errors and model nonlinearities, Pi et al. [11] combined model-based method and kinematics-based method to estimate body sideslip angle. Meanwhile, fuzzy-logic observer was implemented to determine the nonlinear factor and corning stiffness was identified to compensate the tire nonlinearities. The researches above are all based on EKF and its improved form to body sideslip angle and other vehicle state information, but EKF need to calculate state transition matrix by utilizing state function which maybe cause the problem of divergence [12-18].

To address issues associated with the complex calculation of Jacobian matrix and excessive large error caused by system nonlinearity, unscented Kalman filter(UKF) and cubature Kalman filter(CKF) are proposed. Zhou and Qi proposed an adaptive UKF algorithm which consider the impact of noise on the system to estimate body sideslip angle and yaw rate [19]. Similarly, Wang et al. [20] took the relationship of different road noise and sensors noise into account and utilized a road classification method working conjunction with AUKF algorithm. Results showed that the method improves the accuracy of roll rate, pitch rate and sprung mass velocity of vehicle body. In [21], Boada et al. combined adaptive neuro-fuzzy inference system (ANFIS) with UKF to estimate body sideslip angle, which in turn was used to control the vehicle and improves the stability. Moreover, to address the issues caused by tire nonlinearities, Jin and Yin [22] introduced an interacting multiple model (IMM) which consists of two UKF algorithm parts. The simulation in Carsim and Matlab verified the effectiveness of IMM.

Compared to first-order accuracy of Taylor expansion in EKF and second-order accuracy in UKF, CKF can promise three-order accuracy for nonlinear systems. CKF adopts spherical-radial cubature rule to approximate the multidimensional integrals [23-24]. A series of cubature points are used to compute integrals. For UKF, big weight of the sigma point in the center maybe leads to semi-positive and negative definite of covariance matrix, while CKF distribute same weight to sigma points and cubature points, which enhances the numerical stability [25-27]. In [28-30], researchers adopt adaptive law by utilizing innovation information and make use of suboptimal unbiased maximal posterior estimator for system and measurement noise to update covariance in real time. Chen et al. [31] proposed an adaptive CKF algorithm working conjunction with a longitudinal tire force observer to estimate vehicle state information. In this method, an adaptive law was introduced to CKF by setting different weights to innovation vector according to usefulness of information in the slide window.

It is noted that body sideslip angle, yaw rate and lateral tire forces are important for vehicle lateral control. However, most prior methods for vehicle states estimation are based on high-order state vectors which have strong connection with each other. To reduce the risk of semi-positive and negative definite to covariance matrix for CKF, a modular estimation strategy is proposed and applied to estimate vehicle states. Firstly, considering the lateral tire forces nonlinearities, body sideslip angle and yaw rate are preliminarily estimated by ACKF which makes use of innovation information in different tire sideslip angle section. Meanwhile, an adaptive proportion integral observer is introduced to compensate body sideslip angle by utilizing estimated yaw rate. Secondly, a one-order dynamics tire model is adopted to kinematics model. Sum of front lateral force and sum of rear lateral force are estimated by ACKF. In addition, by computing partition coefficient from vertical tire force model, lateral force of ACKF is distributed to four wheels.

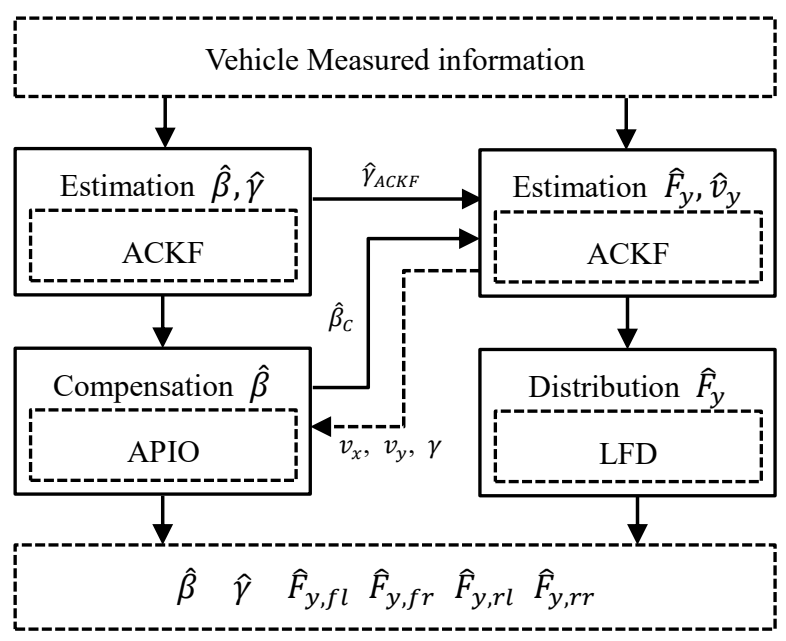

Figure 1 Architecture of the method for state estimation

This paper is organized as follows: Section 2 briefly describes the four-wheel vehicle dynamics model. The proposed observer for body sideslip angle and yaw rate is presented in detail in Section 3. In addition, a new observer for lateral tire force and lateral vehicle speed is discussed in Section 3. Its performance is tested by MATLAB/SimulinkCarsim co-simulation and real vehicle test in Section 4 . 
Section 5 is the conclusion. Section 6 is the declaration.

\section{Four-wheel vehicle dynamics model}

\subsection{Vehicle dynamic model}

The vehicle model presented in this paper is shown in Figure 1. The model is based on the following assumption: 1) neglect the pitch and roll angles of the vehicle and the effect of the suspension. 2) assume that the characteristics of each tire are the same, and the steering system is accurately adjusted, and the left and right front wheels have the same angle of rotation. 3) neglect the effect of vertical rolling resistance. The longitudinal axle of the vehicle is the $x$ axle of xoy coordinate system, and the lateral axle of the vehicle is the $y$ axle. The origin of coordinate system is fixed on the vehicle gravity. The symbols $f l, f r, r l$ and $r r$ are respectively corresponding to the front-left, the front-right, the rear-left and the rear-right. Under the above assumptions, the four-wheel dynamics model can be expressed by the following governing equation

Equation of yaw motion:

$$
\dot{\gamma}=\frac{1}{I_{z}}\left\{\begin{array}{c}
\frac{\left(F_{y, f l} \sin \delta_{f l}-F_{x, f l} \cos \delta_{f l}\right) B_{f}}{2} \\
-\left(F_{x, f l} \sin \delta_{f l}+F_{y, f l} \cos \delta_{f l}\right) l_{f} \\
-\frac{\left(F_{x, f r} \cos \delta_{f r}-F_{y, f r} \sin \delta_{f r}\right) B_{f}}{2} \\
-\left(F_{x, f r} \sin \delta_{f r}+F_{y, f r} \cos \delta_{f r}\right) l_{f} \\
+\frac{\left(F_{x, r r}-F_{y, r l}\right) B_{f}}{2}+\left(F_{y, r r}-F_{y, r l}\right) l_{r}
\end{array}\right\}
$$

Equation of lateral motion:

$$
\begin{aligned}
& a_{y}=\frac{1}{m}\left(\begin{array}{c}
F_{y, f l} \cos \delta_{f l}+F_{y, f r} \cos \delta_{f r}+F_{y, r l} \\
+F_{y, r r}+F_{x, f l} \sin \delta_{f l}+F_{x, f r} \sin \delta_{f r}
\end{array}\right) \\
& =\frac{1}{m} \sum F_{y} \\
& a_{y}=\dot{v}_{y}+v_{x} \gamma
\end{aligned}
$$

Equation of longitudinal motion:

$$
a_{x}=\dot{v}_{x}-v_{y} \gamma
$$

where $F_{y, i j}$ and $F_{x, i j}$ are the lateral tire force and longitudinal tire force $(i=f$ or $r, j=l$ or $r), \gamma$ is the yaw rate, $I_{z}$ represents the moment of inertia, $\delta_{f l}$ and $\delta_{f r}$ are the steering angles of front-left and front-right wheel. $B_{f}$ and $B_{r}$ are the front tread and rear tread of the vehicle. $l_{f}$ and $l_{r}$ are the distances from vehicle gravity center to the front and rear axle. $a_{y}$ stands for the lateral acceleration, $m$ represents the mass, $v_{x}$ is the longitudinal vehicle speed $v_{y}$ is the lateral vehicle speed, $a_{x}$ represents the longitudinal acceleration. $M_{z}$ is moment of vehicle gravity center in xoy-plane.

Four-wheel vehicle dynamics model can distinguish the difference in tire force between the left and right wheels, but the single-track vehicle model pays more attention to the influence of the center of mass slip angle and yaw rate on the vehicle. Therefore, single-track vehicle model is also implemented to estimate the vehicle states when estimating the lateral angle of mass and yaw rate. The dynamics model is shown as follows:

Equation of yaw motion:

$$
\sum M_{z}=F_{y, f} l_{f}-F_{y, r} l_{r}
$$

Equation of lateral motion:

$$
\sum F_{y}=F_{y, f}+F_{y, r} l_{r}
$$

where $F_{y, f}$ is the sum of front-left and front-right lateral tire force, $F_{y, r}$ is the sum of rear-left and rear-right lateral tire force,

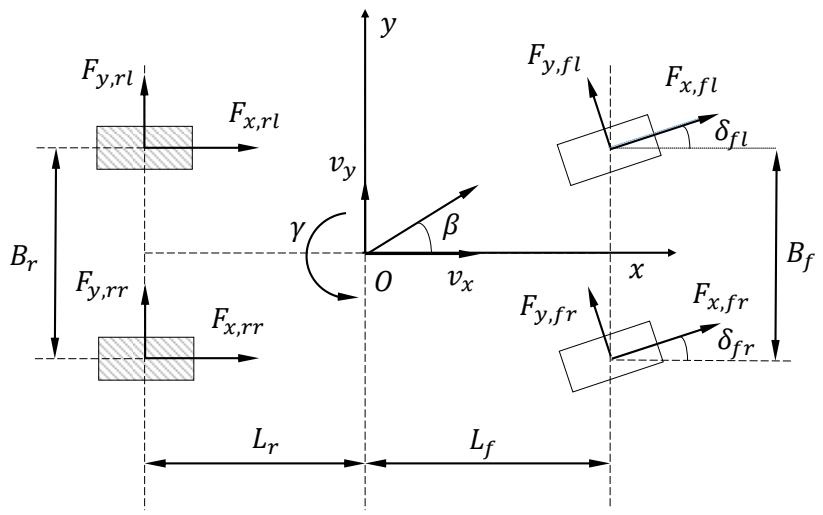

Figure 2 Vehicle dynamic model

The tire sideslip angle $\alpha_{i j}(i=f$ or $r, j=l$ or $r)$ for each wheel can be calculated as: 


$$
\left\{\begin{array}{l}
\alpha_{f l}=\delta_{f l}-\tan ^{-1}\left(\frac{v_{y}+l_{f} \gamma}{v_{x}-0.5 B_{f} \gamma}\right) \\
\alpha_{f r}=\delta_{f l}-\tan ^{-1}\left(\frac{v_{y}+l_{f} \gamma}{v_{x}+0.5 B_{f} \gamma}\right) \\
\alpha_{r l}=-\tan ^{-1}\left(\frac{v_{y}-l_{f} \gamma}{v_{x}-0.5 B_{r} \gamma}\right) \\
\alpha_{r r}=-\tan ^{-1}\left(\frac{v_{y}-l_{f} \gamma}{v_{x}+0.5 B_{r} \gamma}\right)
\end{array}\right.
$$

\subsection{Tire model}

The normal vertical tire force equation $F_{z, i j}(i=$ $f$ or $r, j=l$ or $r$ ) for each wheel can be expressed as:

$$
\left\{\begin{array}{l}
F_{z, f l}=\frac{m g l_{r}}{2\left(l_{f}+l_{r}\right)}-\frac{m h a_{y} l_{r}}{B_{f}\left(l_{f}+l_{r}\right)}+\frac{m a_{x} h}{2\left(l_{f}+l_{r}\right)} \\
F_{z, f r}=\frac{m g l_{r}}{2\left(l_{f}+l_{r}\right)}+\frac{m h a_{y} l_{r}}{B_{f}\left(l_{f}+l_{r}\right)}+\frac{m a_{x} h}{2\left(l_{f}+l_{r}\right)} \\
F_{z, r l}=\frac{m g l_{f}}{2\left(l_{f}+l_{r}\right)}-\frac{m h a_{y} l_{f}}{B_{r}\left(l_{f}+l_{r}\right)}-\frac{m a_{x} h}{2\left(l_{f}+l_{r}\right)} \\
F_{z, r r}=\frac{m g l_{f}}{2\left(l_{f}+l_{r}\right)}+\frac{m h a_{y} l_{f}}{B_{r}\left(l_{f}+l_{r}\right)}-\frac{m a_{x} h}{2\left(l_{f}+l_{r}\right)}
\end{array}\right.
$$

where $g$ is gravitational acceleration, $h$ is the height of vehicle gravity center.

The lateral tire force equation $F_{y, i j}(i=f$ or $r, j=l$ or $r)$ for each wheel can be expressed as:

$$
\left\{\begin{array}{l}
F_{y, f l}=C_{f}\left(\alpha_{f l}, \alpha_{f r}\right) \alpha_{f l} \\
F_{y, f r}=C_{f}\left(\alpha_{f l}, \alpha_{f r}\right) \alpha_{f l} \\
F_{y, r l}=C_{r}\left(\alpha_{r l}, \alpha_{r r}\right) \alpha_{f l} \\
F_{y, r r}=C_{r}\left(\alpha_{r l}, \alpha_{r r}\right) \alpha_{f l}
\end{array}\right.
$$

where $C_{f}\left(\alpha_{f l}, \alpha_{f r}\right)$ and $C_{f}\left(\alpha_{f l}, \alpha_{f r}\right)$ are the front corning stiffness and rear corning stiffness which are related to tire sideslip angle of each wheel. Moreover, the piecewise functions Eq. (10) and Eq. (11) are used to describe the nonlinear characteristics between the lateral tire force and tire sideslip angle [14].

$$
\begin{aligned}
& C_{f}\left(\alpha_{f l}, \alpha_{f r}\right) \\
& = \begin{cases}97000 & \left|\alpha_{f l}\right| \leq 3^{\circ} \text { or }\left|\alpha_{f r}\right| \leq 3^{\circ} \\
90000 & \left|\alpha_{f l}\right|>3^{\circ} \text { or }\left|\alpha_{f r}\right|>3^{\circ}\end{cases} \\
& C_{r}\left(\alpha_{r l}, \alpha_{r r}\right) \\
& =\left\{\begin{array}{lll}
72000 & \left|\alpha_{f l}\right| \leq 3^{\circ} \text { or }\left|\alpha_{f r}\right| \leq 3^{\circ} \\
65000 & \left|\alpha_{f l}\right|>3^{\circ} \text { or } & \left|\alpha_{f r}\right|>3^{\circ}
\end{array}\right.
\end{aligned}
$$

\section{ACKF for Lateral State Information}

The traditional EKF can only estimate the first-order accuracy of the body sideslip angle in the nonlinear region. UKF can achieve second-order accuracy by using the unscented criterion. But CKF can use cubature criterion to improve the estimation accuracy to third order. Therefore, CKF is adopted to estimate vehicle state information. Firstly, Kalman filter is implemented to estimate body sideslip angle and yaw rate. Then, body sideslip angle is corrected by estimated yaw rate, measured longitudinal vehicle speed and lateral vehicle speed via adaptive proportion integral observer(APIO).

\subsection{Extended Kalman Filter}

The continuous-time nonlinear system can be expressed by Eq. (12):

$$
\begin{gathered}
\dot{x}(t)=f(x(t), u(t))+w(t) \\
z(t)=h(x(t))+v(t)
\end{gathered}
$$

where $x$ is the state vector, $u$ is the input vector, $z$ is the measurement vector. $f(*)$ and $h(*)$ are the state function and measurement function, respectively. Moreover, $w$ represents the process noise vector, and $v$ represents the measurement noise vector. $w$ and $v$ are assumed to be zero-mean white satisfies Gaussian distributions, and uncorrelated.

Due to discrete-time characteristic of vehicle motion state information measurement, the above continuous-time nonlinear equation need to be converted to a discrete-time state-space representation. The discrete-time nonlinear system can be expressed by Eq. (13):

$$
\begin{gathered}
x_{k+1}=f_{k}\left(x_{k}, u_{k}\right)+w_{k} \\
z_{k}=h_{k}\left(x_{k}\right)+v_{k}
\end{gathered}
$$

where first-order Euler approximation $\dot{x}_{k+1}=\left(x_{k+1}-\right.$ $\left.x_{k}\right) / t$ is used to discretize the system.

Based on the assumption that a local linearization of system can be an effective description of system nonlinearity, extended Kalman filter can be given by Eq. (13)- (18). Initialization of state $\hat{x}_{0 \mid 0}$ and error covariance $P_{0 \mid 0}$ is given by: 


$$
\begin{gathered}
\hat{x}_{0 \mid 0}=E\left(x_{0 \mid 0}\right) \\
P_{0 \mid 0}=E\left(\left(x_{0 \mid 0}-\hat{x}_{0 \mid 0}\right)\left(x_{0 \mid 0}-\hat{x}_{0 \mid 0}\right)^{T}\right)
\end{gathered}
$$

The priori prediction of state is calculated by:

$$
\hat{x}_{k+1 \mid k}=F_{k+1 \mid k} \hat{x}_{k \mid k}+B_{k \mid k} u_{k \mid k}
$$

where $F_{k+1 \mid k}$ is the state transition matrix, which is the Jacobian matrix of state function $f(*)$ with respect to $x$. $F_{k+1 \mid k}$ is calculated by Eq. (16):

$$
\begin{aligned}
& F_{k+1 \mid k}=\frac{\partial f_{k}\left(x_{k}, u_{k}\right)}{\partial x} \\
& =\left[\begin{array}{cccc}
\frac{\partial f_{1}\left(x_{k}, u_{k}\right)}{\partial x_{1}} & \frac{\partial f_{1}\left(x_{k}, u_{k}\right)}{\partial x_{2}} & \cdots & \frac{\partial f_{1}\left(x_{k}, u_{k}\right)}{\partial x_{n}} \\
\frac{\partial f_{2}\left(x_{k}, u_{k}\right)}{\partial x_{1}} & \frac{\partial f_{2}\left(x_{k}, u_{k}\right)}{\partial x_{2}} & \cdots & \frac{\partial f_{2}\left(x_{k}, u_{k}\right)}{\partial x_{n}} \\
\vdots & \vdots & & \vdots \\
\frac{\partial f_{m}\left(x_{k}, u_{k}\right)}{\partial x_{1}} & \frac{\partial f_{m}\left(x_{k}, u_{k}\right)}{\partial x_{2}} & \cdots & \frac{\partial f_{m}\left(x_{k}, u_{k}\right)}{\partial x_{n}}
\end{array}\right]
\end{aligned}
$$

where $m, n$ represent the dimension of the measurement function $f(*)$ and state vector $x$.

The priori error covariance matrix is given by:

$$
P_{k+1 \mid k}=F_{k+1 \mid k} P_{k \mid k} F_{k+1 \mid k}^{T}+Q_{k}
$$

where $Q_{k}$ is the process noise covariance matrix, and $Q_{k}=\operatorname{cov}\left(w_{i}, w_{j}^{T}\right) . H_{k+1}$ is the state measurement matrix, which is the Jacobian matrix of the measurement function $h(*)$ with respect to $x$. And, $H_{k+1}$ is calculated by Eq. (18):

$$
\begin{aligned}
& H_{k+1}=\frac{\partial h_{k}\left(x_{k}, u_{k}\right)}{\partial x} \\
& =\left[\begin{array}{cccc}
\frac{\partial h_{1}\left(x_{k}, u_{k}\right)}{\partial x_{1}} & \frac{\partial h_{1}\left(x_{k}, u_{k}\right)}{\partial x_{2}} & \cdots & \frac{\partial h_{1}\left(x_{k}, u_{k}\right)}{\partial x_{n}} \\
\frac{\partial h_{2}\left(x_{k}, u_{k}\right)}{\partial x_{1}} & \frac{\partial h_{2}\left(x_{k}, u_{k}\right)}{\partial x_{2}} & \cdots & \frac{\partial h_{2}\left(x_{k}, u_{k}\right)}{\partial x_{n}} \\
\vdots & \vdots & & \vdots \\
\frac{\partial h_{s}\left(x_{k}, u_{k}\right)}{\partial x_{1}} & \frac{\partial h_{s}\left(x_{k}, u_{k}\right)}{\partial x_{2}} & \cdots & \frac{\partial h_{s}\left(x_{k}, u_{k}\right)}{\partial x_{n}}
\end{array}\right]
\end{aligned}
$$

function $h(*)$.

The Kalman gain is updated:

$$
K_{k+1}=P_{k+1 \mid k} H_{k+1}^{T}\left(H_{k+1} P_{k+1 \mid k} H_{k+1}^{T}+R_{k}\right)^{-1}
$$

where $R$ is the measurement noise covariance matrix, and $R_{k}=\operatorname{cov}\left(v_{i}, v_{j}^{T}\right)$.

The posterior prediction of state is computed as:

$$
\hat{x}_{k+1 \mid k+1}=\hat{x}_{k+1 \mid k}+K_{k+1}\left(z_{k+1}-H_{k+1} \hat{x}_{k+1 \mid k}\right)
$$

The posterior error covariance is calculated by:

$$
P_{k+1 \mid k+1}=P_{k+1 \mid k}-K_{k+1} H_{k+1} P_{k+1 \mid k}
$$

\subsection{ACKF for Body Sideslip Angle and Yaw Rate}

CKF is firstly proposed by Canadian researcher Arasaratnam and Haykin to address a typical air-traffic control problem in 2009. The core idea of CKF algorithm is to approximate the posterior mean and covariance of the state by the third-order spherical radial volume criterion for the nonlinear Gaussian system to ensure that the posterior mean and covariance of any nonlinear Gaussian state is approximated by a third-order polynomial in theory.

In the standard CKF algorithm, it is necessary to assume that the statistical characteristics of noise are accurately known. However, in the actual control system, the statistical characteristics of $w_{k}$ and $v_{k}$ are often unknown. The measurement noise is mainly influenced by sensors error which has stable statistical characteristics, but system noise is influenced by system nonlinearities and un-modeled dynamics which are difficultly expressed by constant. Therefore, innovation vector is implemented to estimate the system noise matrix $Q_{k}$. ACKF algorithm is given by Eq. (22)- (30).

1) Initialization equation is the same as Eq. (14).

Time update.

2) Decompose the error covariance matrix by Cholesky decomposition:

$$
P_{k \mid k}=S_{k \mid k} S_{k \mid k}^{T}
$$

3) Calculate cubature points:

where $s$ represents the dimension of the measurement 


$$
\chi_{k \mid k}^{(i)}=S_{k \mid k} \xi_{i}+\hat{x}_{k \mid k} i=1,2, \cdots, L \quad L=2 n
$$

where $\xi_{i}$ is the sigma point, and it is given by Eq. (24)

$$
\xi_{i}=\sqrt{n}\left[\left(\begin{array}{c}
1 \\
0 \\
\vdots \\
0
\end{array}\right) \cdots\left(\begin{array}{c}
0 \\
0 \\
\vdots \\
1
\end{array}\right)\left(\begin{array}{c}
-1 \\
0 \\
\vdots \\
0
\end{array}\right) \quad \cdots\left(\begin{array}{c}
0 \\
0 \\
\vdots \\
-1
\end{array}\right)\right]_{i}
$$

4) Propagate cubature points via the state function $f(*)$ :

$$
\chi_{k \mid k}^{*(i)}=f_{k}\left(\chi_{k \mid k}^{(i)}, u_{k}\right)
$$

5) Calculate the priori prediction matrix of state

$$
\hat{x}_{k+1 \mid k}=\frac{1}{L} \sum_{i=1}^{L} \chi_{k \mid k}^{*(i)}
$$

6) Calculate the priori error covariance matrix

$$
\begin{gathered}
P_{k+1 \mid k}=\frac{1}{L} \sum_{i=1}^{L} \chi_{k \mid k}^{*(i)}\left(\chi_{k \mid k}^{*(i)}\right)^{T}-\hat{x}_{k+1 \mid k}\left(\hat{x}_{k+1 \mid k}\right)^{T} \\
+Q_{k+1}
\end{gathered}
$$

Measurement update.

7) Decompose the priori error covariance matrix by Cholesky decomposition:

$$
P_{k+1 \mid k}=S_{k+1 \mid k} S_{k+1 \mid k}^{T}
$$

8) Calculate cubature points:

$$
\begin{gathered}
\rho_{k+1 \mid k}^{(i)}=S_{k+1 \mid k} \xi_{i}+\hat{x}_{k+1 \mid k} i=1,2, \cdots, L L \\
=2 n
\end{gathered}
$$

9) Propagate cubature points via the measurement function $h(*)$ :

$$
\mathrm{Z}_{k+1 \mid k}^{*(i)}=h_{k}\left(\rho_{k+1 \mid k}^{(i)}, u_{k}\right)
$$

10) Calculate the prediction matrix of measurement:

$$
\hat{z}_{k+1 \mid k}=\frac{1}{L} \sum_{i=1}^{L} \mathrm{Z}_{k+1 \mid k}^{*(i)}
$$

11) Estimate self-correlation covariance matrix:

$$
\begin{gathered}
P_{z z, k+1 \mid k}=\frac{1}{L} \sum_{i=1}^{L} \mathrm{Z}_{k+1 \mid k}^{*(i)}\left(\mathrm{Z}_{k+1 \mid k}^{*(i)}\right)^{T} \\
-\hat{z}_{k+1 \mid k}\left(\hat{z}_{k+1 \mid k}\right)^{T}+R_{k+1}
\end{gathered}
$$

12) Estimate cross-correlation covariance matrix:

$$
\begin{gathered}
P_{x z, k+1 \mid k}=\frac{1}{L} \sum_{i=1}^{L} \chi_{k \mid k}^{(i)}\left(\mathrm{Z}_{k+1 \mid k}^{*(i)}\right)^{T} \\
-\hat{x}_{k+1 \mid k}\left(\hat{z}_{k+1 \mid k}\right)^{T}
\end{gathered}
$$

13) Calculate the Kalman gain:

$$
K_{k+1}=P_{x z, k+1 \mid k}\left(P_{z z, k+1 \mid k}\right)^{-1}
$$

14) Update the posterior prediction of state:

$$
\hat{x}_{k+1 \mid k+1}=\hat{x}_{k+1 \mid k}+K_{k+1}\left(z_{k+1}-\hat{z}_{k+1 \mid k}\right)
$$

15) Update the posterior error covariance matrix:

$$
P_{k+1 \mid k+1}=P_{k+1 \mid k}-K_{k+1} P_{z z, k+1 \mid k} K_{k+1}^{T}
$$

16) Update the system noise covariance matrix:

$$
\begin{gathered}
D_{k}=\sum_{g=f}^{e} e_{k} e_{k}^{T} \\
=\sum_{g=f}^{e}\left(z_{k+1}-\hat{z}_{k+1 \mid k}\right)\left(z_{k+1}-\hat{z}_{k+1 \mid k}\right)^{T} \\
=\sum_{g=1}^{e}\left(z_{k+1}-\hat{z}_{k+1 \mid k}\right)\left(z_{k+1}-\hat{z}_{k+1 \mid k}\right)^{T} \\
-\sum_{g=1}^{f}\left(z_{k+1}-\hat{z}_{k+1 \mid k}\right)\left(z_{k+1}-\hat{z}_{k+1 \mid k}\right)^{T} \\
Q_{k}=\frac{\varepsilon K_{k} D_{k} K_{k}^{T}+(\sigma-\varepsilon) Q_{0}}{\sigma}
\end{gathered}
$$

Combining Eq. (1) - (6) and Eq. (9) - (11), the state space model and measurement equation are expressed by Eq. (39) and Eq. (40), respectively. 


$$
\begin{gathered}
\left\{\begin{array}{c}
\dot{\beta}=-\frac{C_{f}+C_{r}}{m v_{x}} \beta+\left(\frac{\left(C_{r} l_{r}-C_{f} l_{f}\right)}{m v_{x}^{2}}-1\right) \gamma+\frac{C_{f}}{m v_{x}} \delta \\
\dot{\gamma}=\frac{-l_{f} C_{f}+l_{r} C_{r}}{I_{Z Z}} \beta-\frac{\left(C_{r} l_{r}^{2}+C_{f} l_{f}^{2}\right)}{I_{Z Z} v_{x}} \gamma+\frac{l_{f} C_{f}}{I_{Z Z}} \delta
\end{array}\right. \\
\left\{\begin{array}{c}
a_{y}=-\frac{l_{f} C_{f}+l_{r} C_{r}}{m} \beta-\frac{\left(C_{r} l_{r}-C_{f} l_{f}\right)}{m} \gamma+\frac{C_{f}}{m} \delta \\
\gamma=\gamma
\end{array}\right.
\end{gathered}
$$

The state vector $x=\left[\begin{array}{ll}\beta & \gamma\end{array}\right]^{T}$, the measurement vector $z=\left[\begin{array}{ll}a_{y} & \gamma\end{array}\right]^{T}$.

\subsection{Correction by Adaptive Proportion Integral Observer}

In order to observe the body sideslip angle accurately, compensation algorithm is proposed to implemented and it estimates the body sideslip angle utilizing estimated yaw rate. The APIO is given by Eq. (41).

$$
\begin{gathered}
\beta_{C}=k_{p}\left(\frac{\hat{v}_{y}}{v_{x}}-\hat{\beta}_{A C K F}\right)+k_{i} \int\left(\frac{\hat{v}_{y}}{v_{x}}-\hat{\beta}_{A C K F}\right) \\
+\hat{\beta}_{A C K F}
\end{gathered}
$$

where $\hat{v}_{y}$ represents the estimated lateral vehicle speed, and $v_{x}$ represents the longitudinal vehicle speed measured by sensors. $\hat{\beta}_{A C K F}$ stands for the body sideslip angle estimated by ACKF. $k_{p}$ and $k_{i}$ represent the proportion gain and integration gain, respectively. $k_{p}$ and $k_{i}$ is given by Eq. (41) and (42).

$$
\begin{aligned}
& k_{p}=k_{1} e^{k_{2}\left(\gamma_{m}-\widehat{\gamma}_{A C K F}\right)} \\
& k_{i}=k_{3} e^{k_{4}\left(\gamma_{m}-\widehat{\gamma}_{A C K F}\right)}
\end{aligned}
$$

where $\gamma_{m}$ and $\hat{\gamma}_{A C K F}$ are the yaw rate measured by the sensor and estimated by ACKF, respectively. $k_{1}, k_{2}, k_{3}$ and $k_{4}$ are the correlation coefficients and their limit of values are $0 \leq k_{1} \leq 0.5,0 \leq k_{2} \leq 0.5,0 \leq k_{3} \leq 0.5$ and $0 \leq k_{4} \leq 0.5$.

\subsection{ACKF for Lateral Tire Force}

In order to estimate the lateral tire force, ACKF algorithm is adopted. A widely used empirical tire model is mainly based on empirical formulations deriving from tire test data and an amount of parameters [32]. To avoid complex calculation and utilizing tire test data, linearized tire force models is adopted. Based on the bicycle model and one-order dynamics tire model [33], state space model for lateral tire force can be built. First of all, the lateral tire forces can be linearly approximated and are given as:

$$
\begin{gathered}
F_{y, f}=-2 C_{f} \alpha_{f}=-2\left(\beta+\frac{\gamma l_{f}}{v_{x}}-\delta\right) \\
F_{y, r}=-2 C_{r} \alpha_{r}=-2\left(\beta-\frac{\gamma l_{r}}{v_{x}}\right)
\end{gathered}
$$

where $\beta$ is the body sideslip angle estimated by APIO, and $\gamma$ is the yaw rate estimated by ACKF. In order to illustrate the transient behavior of the tire, a typical dynamic model, the first-order dynamic model is used, which is expressed as follows:

$$
\tau_{\text {lag }, i} \dot{F}_{y, i}+F_{y, i}=\bar{F}_{y, i}
$$

where $\tau_{\text {lag, } i}$ is relaxation time constant, defined as a constant associated with vehicle speed. $\bar{F}_{y, i}$ is the lateral tire force calculated by Eq. (44) and (45).By combining Eq (44) - (46), the dynamic lateral tire force equation can be computed as:

$$
\begin{gathered}
\dot{F}_{y, f}=-\frac{F_{y, f}}{\tau_{\text {lag }, f}}-\frac{2 C_{f}}{\tau_{\text {lag }, f}} \beta-\frac{2 C_{f} l_{f}}{\tau_{\text {lag }, f} v_{x}} \gamma+\frac{2 C_{f}}{\tau_{\text {lag }, f}} \delta \\
\dot{F}_{y, r}=-\frac{F_{y, r}}{\tau_{\text {lag }, r}}-\frac{2 C_{r}}{\tau_{\text {lag }, r}} \beta+\frac{2 C_{r} l_{r}}{\tau_{\text {lag }, f} v_{x}} \gamma
\end{gathered}
$$

Meanwhile, $v_{y}$ is also estimated and can be expressed by:

$$
\dot{v}_{y}=\frac{F_{y, r}}{m}+\frac{F_{y, f}}{m}-v_{x} \gamma
$$

The lateral acceleration $a_{y}$ can be measured by the sensor. Therefore it is suitable to play an role as measurement vector and $a_{y}$ is calculated by:

$$
a_{y}=\frac{F_{y, r}}{m}+\frac{F_{y, f}}{m}
$$

According to Eq. (47)- (50), state space model is built. The state vector $x_{2}=\left[\begin{array}{lll}F_{y, f} & F_{y, r} & v_{y}\end{array}\right]^{T}$. The input are $\delta, v_{x}$, $\beta$ and $\gamma$.The measurement vector is $z_{2}=a_{y}$. Moreover, the state function $f_{2}\left(x_{2}\right)$ is given by: 


$$
\begin{aligned}
& f_{2}\left(x_{2}\right) \\
& =\left(\begin{array}{c}
-\frac{x_{2}(1)}{\tau_{\text {lag }, f}}-\frac{2 C_{f}}{\tau_{\text {lag }, f}} \beta-\frac{2 C_{f} l_{f}}{\tau_{\text {lag }, f} v_{x}} \gamma+\frac{2 C_{f}}{\tau_{\text {lag }, f}} \delta \\
-\frac{x_{2}(2)}{\tau_{\text {lag }, r}}-\frac{2 C_{r}}{\tau_{\text {lag }, r}} \beta+\frac{2 C_{r} l_{r}}{\tau_{\text {lag }, f} v_{x}} \gamma \\
\frac{x_{2}(1)}{m}+\frac{x_{2}(2)}{m}-v_{x} \gamma
\end{array}\right)_{3 \times 1}
\end{aligned}
$$

The measurement function $h_{2}\left(x_{2}\right)$ is given by:

$$
h_{2}\left(x_{2}\right)=\left(\frac{x_{2}(1)}{m}+\frac{x_{2}(2)}{m}\right)
$$

ACKF is used to estimate $F_{y, f}, F_{y, r}$ and $v_{y}$, but the lateral tire forces of four wheels need to be estimated. Hence, by utilizing vertical model, the partition coefficient is calculated and the lateral tire forces of four wheels are computed as:

$$
\left\{\begin{aligned}
\hat{F}_{y, f l} & =\frac{F_{z, f l}}{F_{z, f l}+F_{z, f r}} \hat{F}_{y, f} \\
\hat{F}_{y, f r} & =\frac{F_{z, f r}}{F_{z, f l}+F_{z, f r}} \hat{F}_{y, f} \\
\hat{F}_{y, r l} & =\frac{F_{z, r l}}{F_{z, r l}+F_{z, r r}} \hat{F}_{y, r} \\
\hat{F}_{y, r r} & =\frac{F_{z, r r}}{F_{z, r l}+F_{z, r r}} \hat{F}_{y, r}
\end{aligned}\right.
$$

\section{Simulation and Experiments}

To evaluate the performance of the proposed estimation method in this paper, the simulations have been carried out in the Matlab/Simulink and CarSim software. The estimation algorithms are designed in the Matlab/Simulink, and the vehicle model in CarSim is achieved. CarSim contains a variety of vehicle models with up to 27 degrees of freedom, rich in parameter variables, and can realize the virtual test of cars in various scenarios. What is more, CarSim provides the reference data for comparison with the proposed method. Two simulation tests are employed to demonstrate the proposed method. The first is a double lane change maneuver of a vehicle traveling at $80 \mathrm{~km} / \mathrm{h}$ on a road surface with friction coefficients of 0.85 . The second is a sine steer maneuver of a vehicle traveling at $48 \mathrm{~km} / \mathrm{h}$ on a road surface

\begin{tabular}{|c|c|c|}
\hline Parameters & Symbol & Value and Units \\
\hline Vehicle mass & $m$ & $1900 \mathrm{~kg}$ \\
\hline $\begin{array}{l}\text { Distances from vehicle gravity center to } \\
\text { the front axle }\end{array}$ & $l_{f}$ & $1.25 m$ \\
\hline $\begin{array}{l}\text { Distances from vehicle gravity center to } \\
\text { the rear axle }\end{array}$ & $l_{r}$ & $1.53 m$ \\
\hline Height of gravity center & $h$ & $0.52 m$ \\
\hline Treads of the front wheels & $B_{f}$ & $1.50 \mathrm{~m}$ \\
\hline Treads of the rear wheels & $B_{r}$ & $1.51 \mathrm{~m}$ \\
\hline $\begin{array}{l}\text { Equivalent cornering stiffness of front } \\
\text { wheel }\end{array}$ & $C_{f}$ & $97000 \mathrm{~N} / \mathrm{rad}$ \\
\hline $\begin{array}{l}\text { Equivalent cornering stiffness of rear } \\
\text { wheel }\end{array}$ & $C_{r}$ & $72000 \mathrm{~N} / \mathrm{rad}$ \\
\hline Effective radius of wheel & $\mathrm{r}$ & $0.3284 m$ \\
\hline Moment of inertia & $I_{z}$ & $2904.8 \mathrm{~kg} \cdot \mathrm{m}^{2}$ \\
\hline
\end{tabular}
with friction coefficients of 0.85 . The relevant parameters of the vehicle model are shown in Table 1.

Table 1 Parameters of the test vehicle in CarSim

\subsection{Double lane change maneuver with longitudinal speed at $80 \mathrm{~km} / \mathrm{h}$}

To accurately evaluate the performance of the proposed methods, mean absolute error(MAE) and root mean square error(RMSE) are utilized, which are calculated as follows:

$$
\begin{aligned}
\text { MAE } & =\frac{1}{n} \sum_{i=0}^{n} S_{\text {actual }, i}-S_{\text {estimated }, i} \\
\text { RMSE } & =\sqrt{\frac{1}{n} \sum_{i=0}^{n}\left(S_{\text {actual }, i}-S_{\text {estimated }, i}\right)^{2}}
\end{aligned}
$$

where $S_{\text {actual }}$ represents the measured signal or the data from Carsim, $S_{\text {estimated }}$ represents the estimated signal, and $n$ is the number of the samples during the simulation or the test.

As shown in Fig. 3, the longitudinal vehicle speed is about $80 \mathrm{~km} / \mathrm{h}$ and steering wheel angle is about from $-110^{\circ}$ to $100^{\circ}$. Fig. 4 shows the lateral vehicle speed and lateral acceleration. During the maneuver, the lateral vehicle speed is between $-4 \mathrm{~km} / \mathrm{h}$ and $4 \mathrm{~km} / \mathrm{h}$ and the lateral acceleration is about from $-8 \mathrm{~m} / \mathrm{s}^{2}$ to $8 \mathrm{~m} / \mathrm{s}^{2}$, which means that tires state could be in the nonlinear regions. 


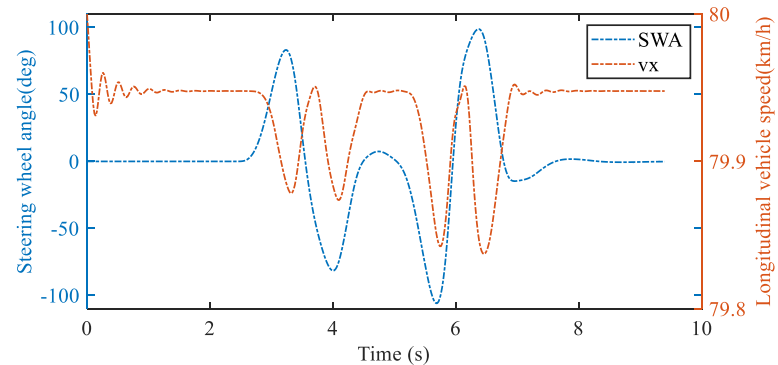

Figure 3 The steering wheel angle and longitudinal vehicle speed

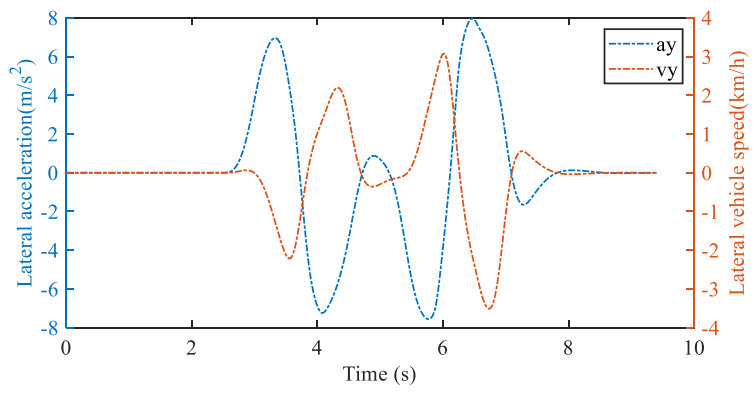

Figure 4 The lateral acceleration and lateral vehicle speed

As shown in Fig. 5, the body sideslip angle estimated by ACKF is more accurate than EKF and CKF. Furthermore, APIO is utilized to compensate the error caused by the nonlinearity of vehicle model and it is demonstrated that the body sideslip angle adjusted by APIO is closer to the measured values. Table 2 shows the MAE and RMSE of the body sideslip angle. MAE and RMSE of EKF is $-3.008 \mathrm{e}-4$ rad and 3.350e-3 rad, respectively, while MAE and RMSE of ACKF with APIO are smaller than those of EKF, which are $-1.145 \mathrm{e}-4 \mathrm{rad}$ and $1.421 \mathrm{e}-3 \mathrm{rad}$, respectively.

Fig. 6 shows the estimated values and actual values of yaw rate, and the proposed algorithm can estimate the yaw rate more accurately than EKF. It can be found in Table 2 that the accuracy of the estimated yaw rate is satisfactory and the MAE and RMSE are $-2.014 \mathrm{e}-3 \mathrm{rad} / \mathrm{s}$ and $3.479 \mathrm{e}-2 \mathrm{rad} / \mathrm{s}$, respectively and that of EKF are $-2.310 \mathrm{e}-3 \mathrm{rad} / \mathrm{s}$ and $3.557 \mathrm{e}-$ $2 \mathrm{rad} / \mathrm{s}$, respectively. The estimation precision of EKF is slightly worse than that of the proposed ACKF.

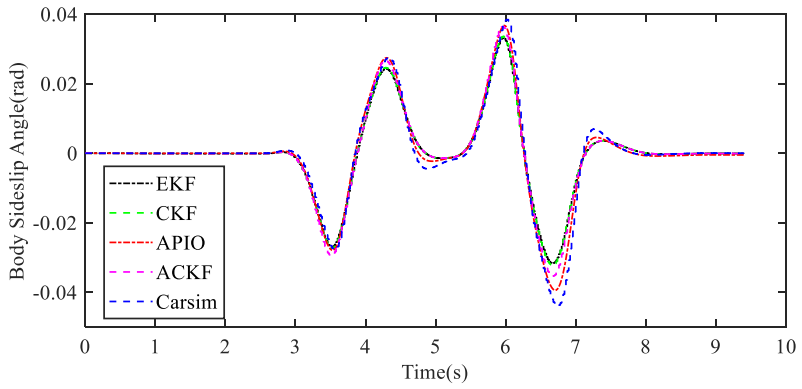

Figure 5 The body sideslip angle estimated by ACKF and EKF

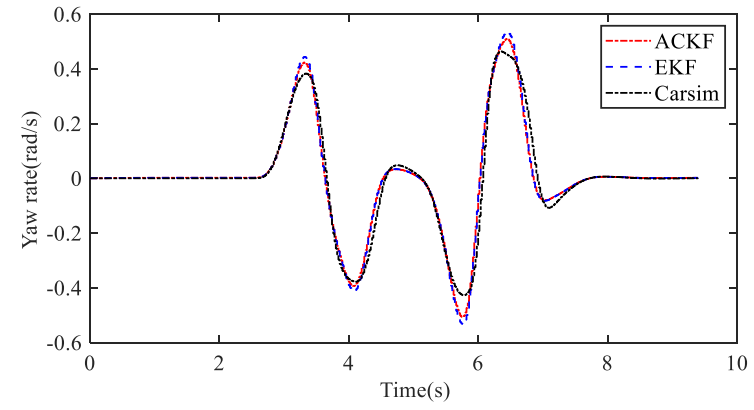

Figure 6 The yaw rate estimated by ACKF and EKF

Table 2 Errors of the body sideslip angle and yaw rate at DLC80

\begin{tabular}{lcc}
\hline & EKF & ACKF+APIO \\
\hline MAE of the body sideslip angle & $-3.008 \mathrm{e}-4$ & $-1.145 \mathrm{e}-4$ \\
RMSE of the body sideslip angle & $3.350 \mathrm{e}-3$ & $1.421 \mathrm{e}-3$ \\
MAE of the yaw rate & $-2.310 \mathrm{e}-3$ & $-2.014 \mathrm{e}-3$ \\
RMSE of the yaw rate & $3.557 \mathrm{e}-2$ & $3.479 \mathrm{e}-2$ \\
\hline
\end{tabular}

As shown in Fig. 7, the tire lateral forces of four wheels are estimated by two methods and ACKF has a higher accuracy than EKF. As shown in Table 3, MAE of tire lateral forces of four wheels estimated by EKF are $-60.411 \mathrm{~N}$, $52.583 \mathrm{~N},-9.484 \mathrm{~N}$ and $-10.254 \mathrm{~N}$, respectively, while those of ACKF are $-81.153 \mathrm{~N},-0.8911 \mathrm{~N},-70.255 \mathrm{~N}$ and $90.446 \mathrm{~N}$, respectively. In addition, RMSE of tire lateral forces of four wheels estimated by EKF are $541.399 \mathrm{~N}, 576.519 \mathrm{~N}$, $513.450 \mathrm{~N}$ and $549.069 \mathrm{~N}$, respectively. Compared to EKF's error, RMSE of ACKF is $319.104 \mathrm{~N}, 282.539 \mathrm{~N}, 329.104 \mathrm{~N}$ and $340.056 \mathrm{~N}$, respectively. Although MAE of EKF is smaller than that of ACKF, RMSE of EKF is at least 1.5 times larger than that of ACKF, that is because EKF has 
greater volatility than ACKF, which is not suitable for vehicle system.

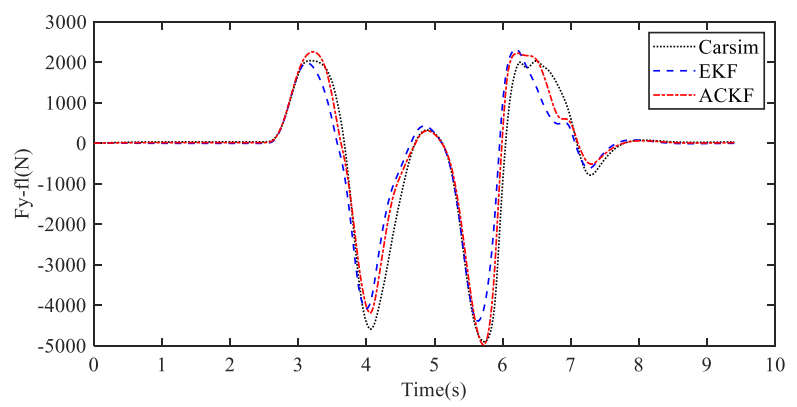

(a)

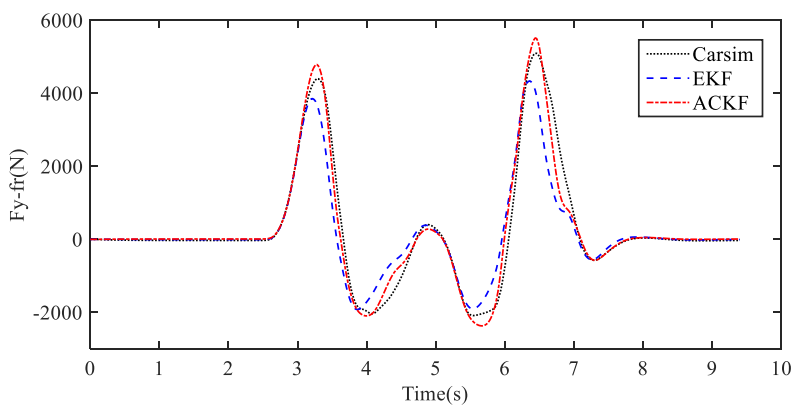

(b)

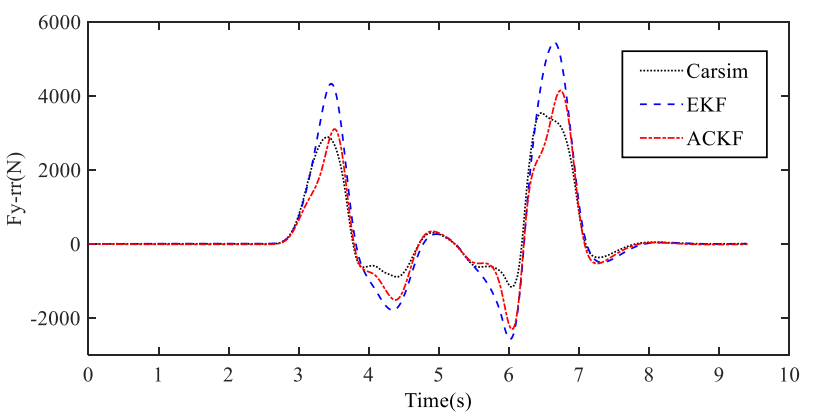

(c)

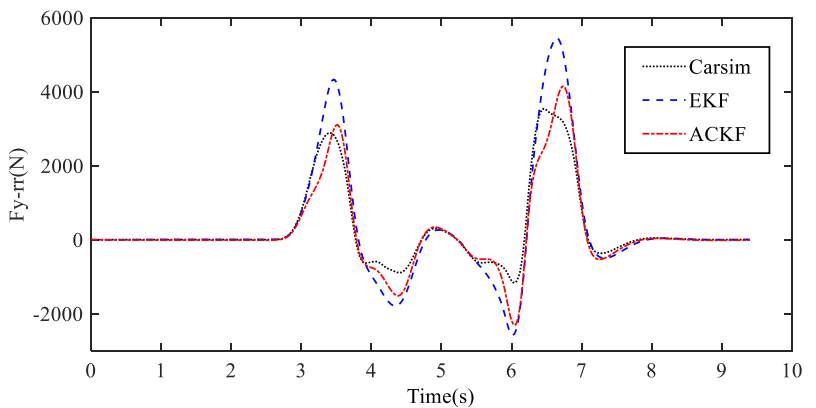

(d)

Figure 7 The lateral tire forces estimation in the simulation: (a) front left wheel, (b) front right wheel, (c) rear left wheel, (d) rear right wheel.

Table 3 Errors of the lateral tire force of four wheels at DLC80

\begin{tabular}{lcc}
\hline & EKF & ACKF \\
\hline MAE of the front left wheel & -60.411 & -81.153 \\
RMSE of the front left wheel & 541.399 & 319.104 \\
MAE of the front right wheel & 52.583 & -0.8911 \\
RMSE of the front right wheel & 576.519 & 282.539 \\
MAE of the rear left wheel & -9.484 & -70.255 \\
RMSE of the rear left wheel & 513.450 & 329.104 \\
MAE of the rear right wheel & -10.254 & 90.446 \\
RMSE of the rear right wheel & 549.069 & 340.056 \\
\hline
\end{tabular}

\subsection{Sine steer maneuver with longitudinal speed at $\mathbf{5 0}$ $\mathrm{km} / \mathrm{h}$}

As shown in Fig. 8, the longitudinal vehicle speed is about $50 \mathrm{~km} / \mathrm{h}$ and steering wheel angle is about from $-100^{\circ}$ to $100^{\circ}$. Fig. 9 shows the lateral vehicle speed and lateral acceleration. During the maneuver, the lateral vehicle speed is between $-1 \mathrm{~km} / \mathrm{h}$ and $1 \mathrm{~km} / \mathrm{h}$ and the lateral acceleration is about from $-5 \mathrm{~m} / \mathrm{s}^{2}$ to $5 \mathrm{~m} / \mathrm{s}^{2}$.

As shown in Fig. 10, the body sideslip angle estimated by ACKF is more accurate than EKF and CKF. Table 4 shows the MAE and RMSE of the body sideslip angle. MAE and RMSE of EKF are -5.711e-4 rad and 3.088e-3 rad respectively, while MAE and RMSE of ACKF with APIO are smaller than those of EKF, which are -3.081e-4 rad and $2.319 \mathrm{e}-3$ rad respectively.

Fig. 11 shows the estimated values and actual values of yaw rate, and the results obtained from proposed algorithm 
can estimate the yaw rate more accurately than EKF as shown in Fig. 11. It can be found in Table 4 that the accuracy of the estimated yaw rate is satisfactory and the MAE and RMSE are $-3.653 \mathrm{e}-3 \mathrm{rad} / \mathrm{s}$ and $1.327 \mathrm{e}-2 \mathrm{rad} / \mathrm{s}$, respectively and that of EKF are $-3.717 \mathrm{e}-3 \mathrm{rad} / \mathrm{s}$ and $5.484 \mathrm{e}-2 \mathrm{rad} / \mathrm{s}$, respectively. The estimation precision of EKF is worse than that of the proposed ACKF.

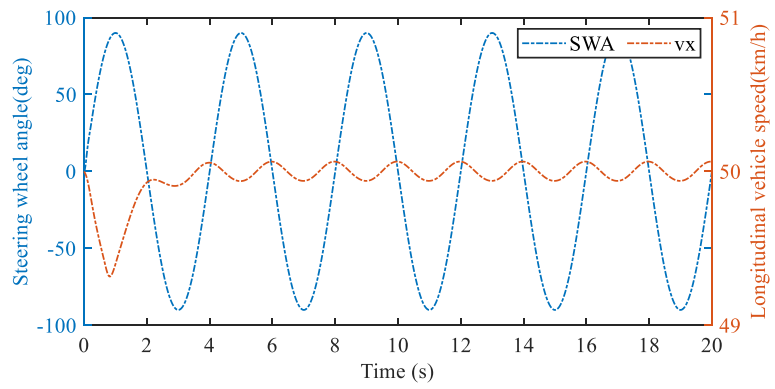

Figure 8 The steering wheel angle and longitudinal vehicle speed

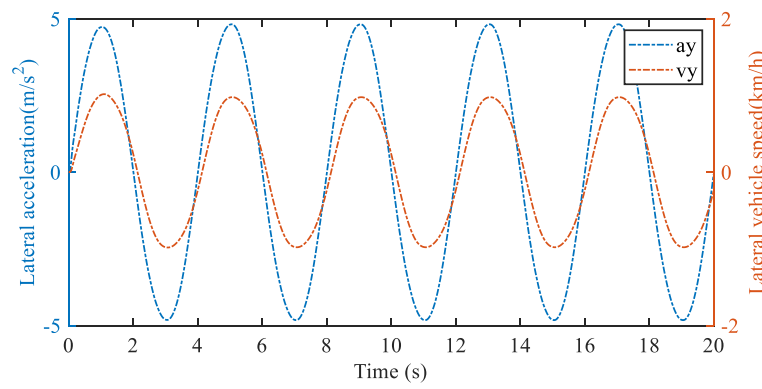

Figure 9 The lateral acceleration and lateral vehicle speed

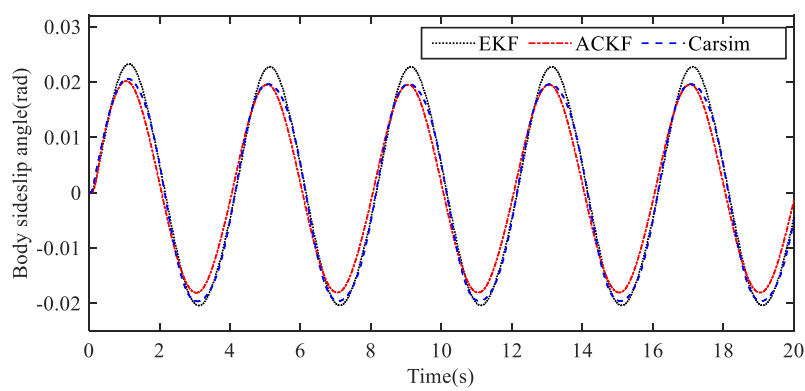

Figure 10 The body sideslip angle estimated by ACKF and EKF

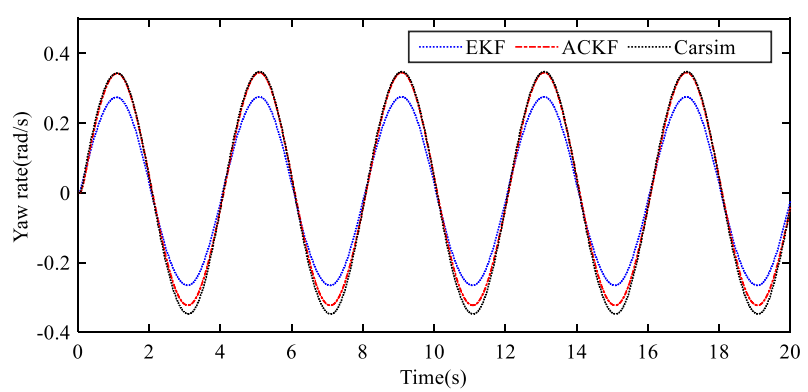

Figure 11 The yaw rate estimated by ACKF and EKF

Table 4 Errors of the body sideslip angle and yaw rate at SIN50

\begin{tabular}{lcc}
\hline & EKF & ACKF \\
\hline MAE of the body sideslip angle & $-5.711 \mathrm{e}-4$ & $-3.081 \mathrm{e}-4$ \\
RMSE of the body sideslip angle & $3.088 \mathrm{e}-3$ & $2.319 \mathrm{e}-3$ \\
MAE of the yaw rate & $-3.717 \mathrm{e}-3$ & $-3.653 \mathrm{e}-3$ \\
RMSE of the yaw rate & $5.484 \mathrm{e}-2$ & $1.327 \mathrm{e}-2$ \\
\hline
\end{tabular}

As shown in Fig. 12, the tire lateral forces of four wheels are estimated by two methods and ACKF has a higher accuracy than EKF.As shown in Table 5, MAE of tire lateral forces of four wheels estimated by EKF are $-14.123 \mathrm{~N}$, $121.758 \mathrm{~N},-65.707 \mathrm{~N}$ and $141.341 \mathrm{~N}$, respectively, while those of ACKF are $20.436 \mathrm{~N}, 19.959 \mathrm{~N}, 12.180 \mathrm{~N}$ and 87.824 $\mathrm{N}$, respectively. In addition, RMSE of tire lateral forces of four wheels estimated by EKF are $232.309 \mathrm{~N}, 238.642 \mathrm{~N}$, $283.593 \mathrm{~N}$ and $337.465 \mathrm{~N}$, respectively. Compared to EKF's error, RMSE of ACKF is $56.879 \mathrm{~N}, 57.205 \mathrm{~N}, 53.755 \mathrm{~N}$ and $110.606 \mathrm{~N}$, respectively. Although MAE of front left lateral tire force by EKF is smaller than that of ACKF, MAE of front right, rear left and rear right lateral tire forces by EKF are larger than those of ACKF and RMSE of EKF is at least 3 times larger than that of ACKF. Therefore, it is illustrated that estimation by ACKF is more accurate than EKF.

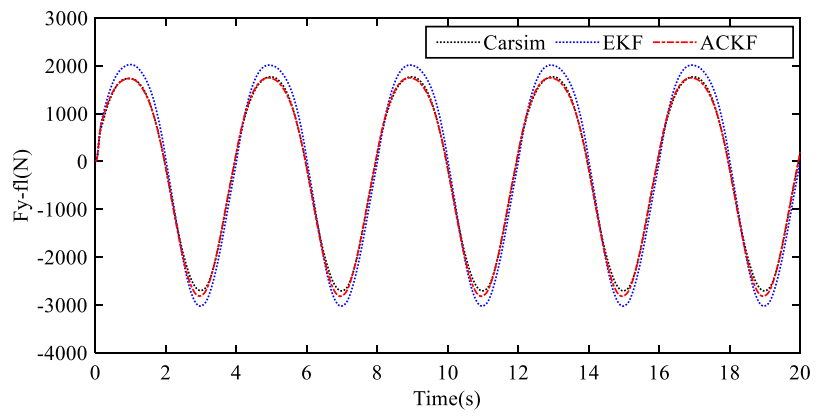

(a) 


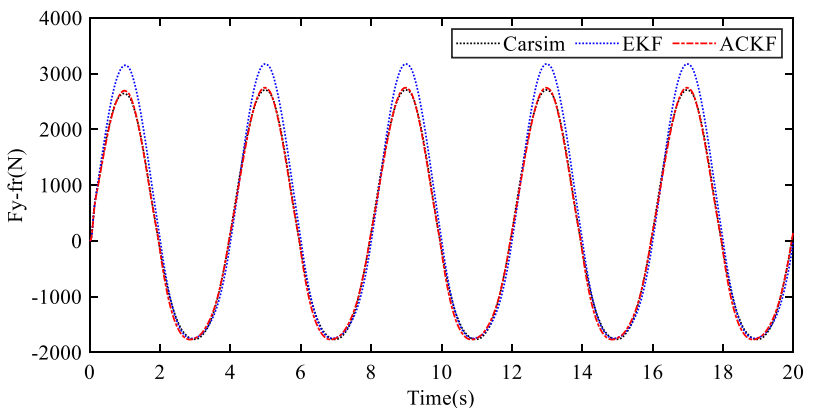

(b)

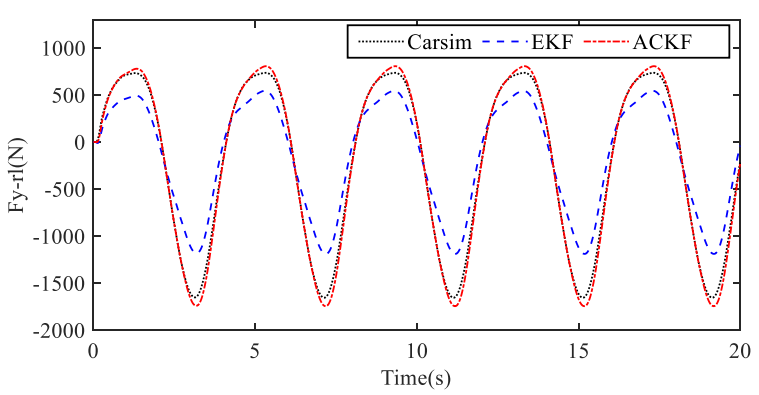

(c)

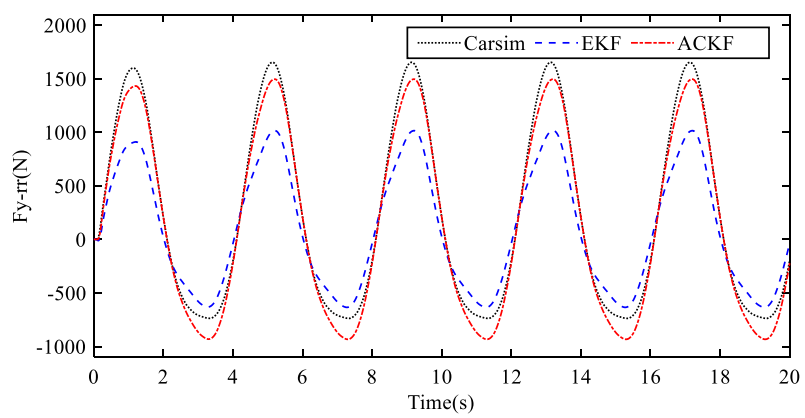

(d)

Figure 12 The lateral tire forces estimation in the simulation: (a) front left wheel, (b) front right wheel, (c) rear left wheel, (d) rear right wheel.

Table 5 Errors of the lateral tire force of four wheels at SIN50

\begin{tabular}{lcc}
\hline & EKF & ACKF \\
\hline MAE of the front left wheel & -14.123 & 20.436 \\
RMSE of the front left wheel & 232.309 & 56.879 \\
MAE of the front right wheel & -121.758 & 19.959 \\
RMSE of the front right wheel & 238.642 & 57.205 \\
MAE of the rear left wheel & -65.707 & 12.180 \\
\hline
\end{tabular}

\begin{tabular}{lll}
\hline RMSE of the rear left wheel & 283.593 & 53.755 \\
MAE of the rear right wheel & 141.341 & 87.824 \\
RMSE of the rear right wheel & 337.465 & 110.606 \\
\hline
\end{tabular}

\subsection{Car test verification}

In order to better verify the effect in the real car, the experiment was carried out. The vehicle test system is shown in Fig. 13. The car used in the test is a sport utility vehicle. ABDynamics steering robot is a computer-controlled vehicle robot test product, which is specially used for dangerous condition of vehicle dynamics test. It can learn a path by manual driving, and then create this path from the recorded data. Dual antenna sensor is a GPS data acquisition system with high accuracy and the ability to measure longitudinal speed, lateral speed and sideslip angle at $100 \mathrm{~Hz}$. Longitudinal and lateral acceleration can be measured by IMU. The collected data can be output in various forms such as CAN, RS232, analog and digital. CAN bus output includes: speed, sideslip angle, pitch angle and roll angle and yaw rate. Steering angle sensor, wheel speed sensor and cylinder pressure sensor can measure the steering wheel angle, wheel speed and cylinder pressure. Wheel speed and cylinder pressure is used for early correction of other signals.

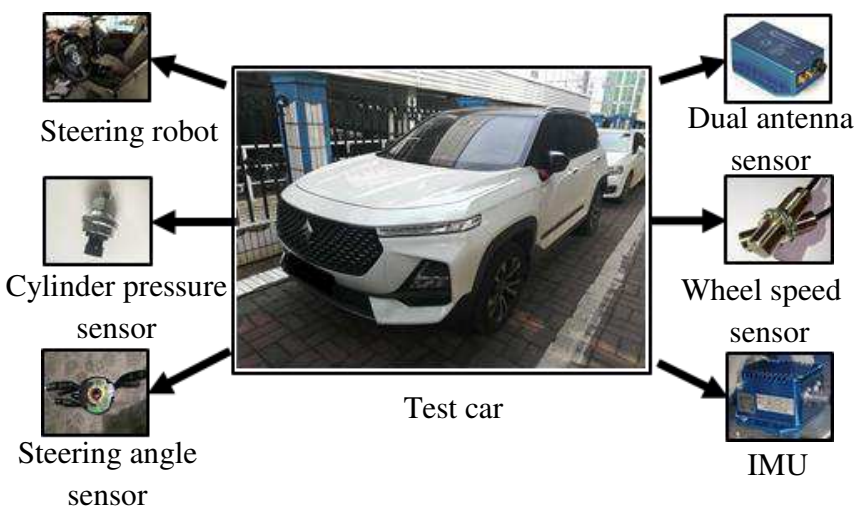

Figure 13 Test system

As shown in Fig. 14, the longitudinal vehicle speed is range from $10 \mathrm{~km} / \mathrm{h}$ to $40 \mathrm{~km} / \mathrm{h}$. and steering wheel angle varies about from $-120^{\circ}$ to $130^{\circ}$. Fig. 15 shows the lateral vehicle speed and lateral acceleration. During the maneuver, the lateral vehicle speed is between $-1 \mathrm{~km} / \mathrm{h}$ and $1 \mathrm{~km} / \mathrm{h}$ and the lateral acceleration is about from $-5 \mathrm{~m} / \mathrm{s}^{2}$ to $5 \mathrm{~m} / \mathrm{s}^{2}$. 


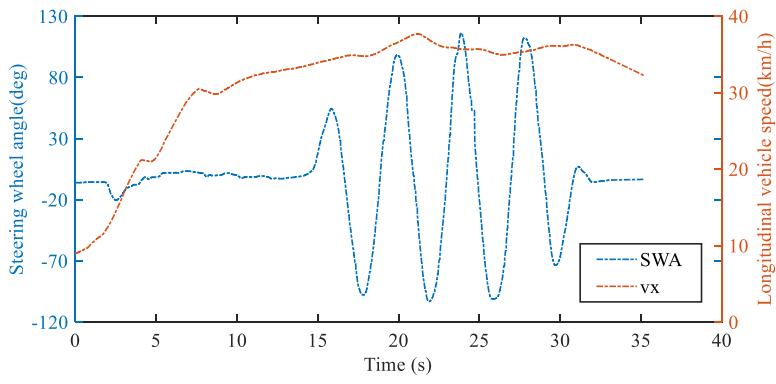

Figure 14 The steering wheel angle and longitudinal vehicle speed

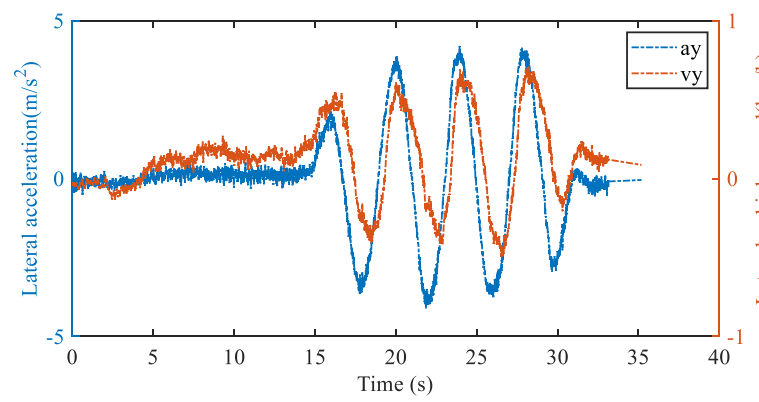

Figure 15 The lateral acceleration and lateral vehicle speed

As shown in Fig. 16, the body sideslip angle estimated by $\mathrm{ACKF}+\mathrm{APIO}$ is more accurate than EKF and ACKF. Table 6 shows the MAE and RMSE of the body sideslip angle. MAE and RMSE of EKF are 4.0340e-3 rad and 1.004e-2 rad respectively, while MAE and RMSE of ACKF with APIO are smaller than those of EKF, which are 6.003e-4 rad and 3.197e-3 rad respectively.

Fig. 17 shows the estimated values and actual values of yaw rate, and the proposed algorithm can estimate the yaw rate more accurately than EKF as shown in Fig. 17. It can be found in Table 6 that the accuracy of the estimated yaw rate is satisfactory and the MAE and RMSE are $1.315 \mathrm{e}-3 \mathrm{rad} / \mathrm{s}$ and $5.867 \mathrm{e}-3 \mathrm{rad} / \mathrm{s}$, respectively and that of EKF are -7.371e$3 \mathrm{rad} / \mathrm{s}$ and $3.837 \mathrm{e}-2 \mathrm{rad} / \mathrm{s}$, respectively. The estimation precision of EKF is worse than that of the proposed ACKF.

Due to the lack of the measurement equipment of lateral tire force, it is difficult to show the reference of the tire force. However, lateral tire force estimation is still shown in Fig. 18.

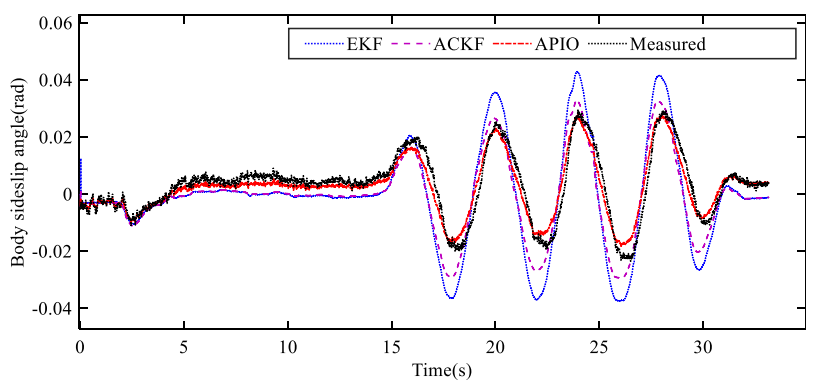

Figure 16 The body sideslip angle estimated by ACKF and EKF

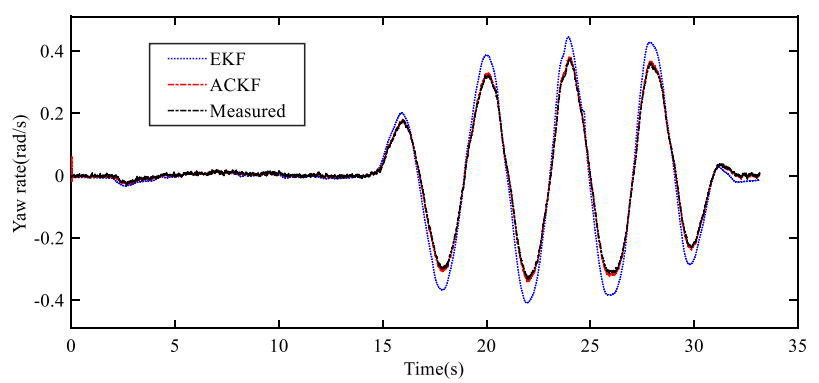

Figure 17 The yaw rate estimated by ACKF and EKF

Table 6 Errors of the body sideslip angle and yaw rate at car test

\begin{tabular}{lcc}
\hline & EKF & ACKF+APIO \\
\hline MAE of the body sideslip angle & $4.0340 \mathrm{e}-3$ & $6.003 \mathrm{e}-4$ \\
RMSE of the body sideslip angle & $1.004 \mathrm{e}-2$ & $3.197 \mathrm{e}-3$ \\
MAE of the yaw rate & $7.371 \mathrm{e}-3$ & $1.315 \mathrm{e}-3$ \\
RMSE of the yaw rate & $3.837 \mathrm{e}-2$ & $5.867 \mathrm{e}-3$ \\
\hline
\end{tabular}

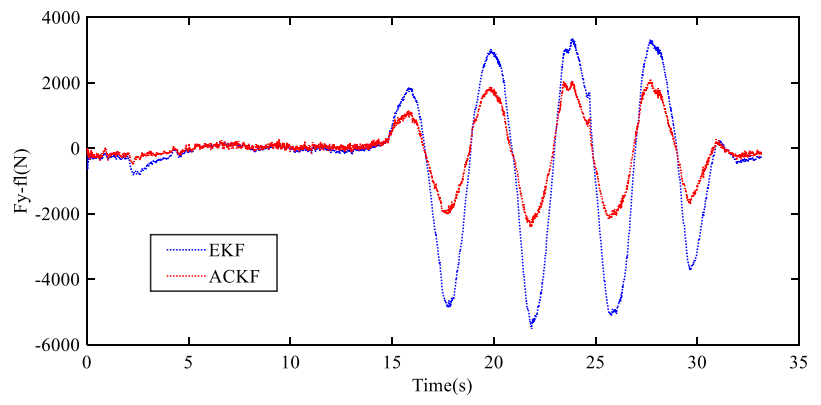

(a) 


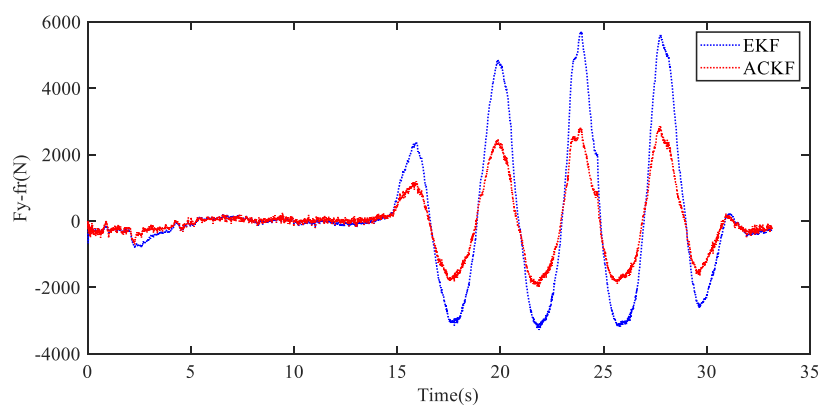

(b)

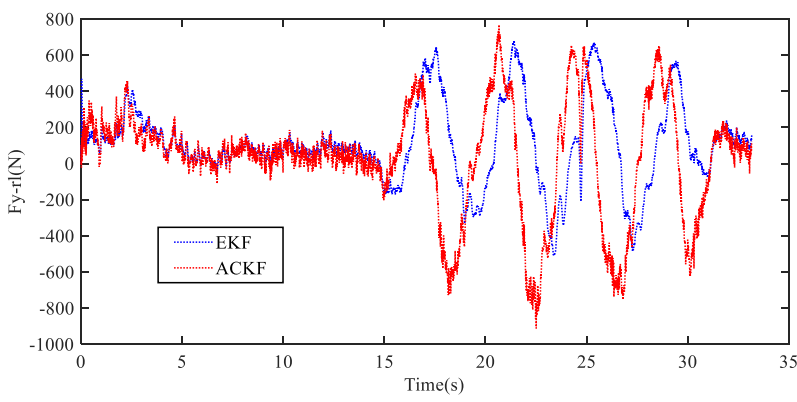

(c)

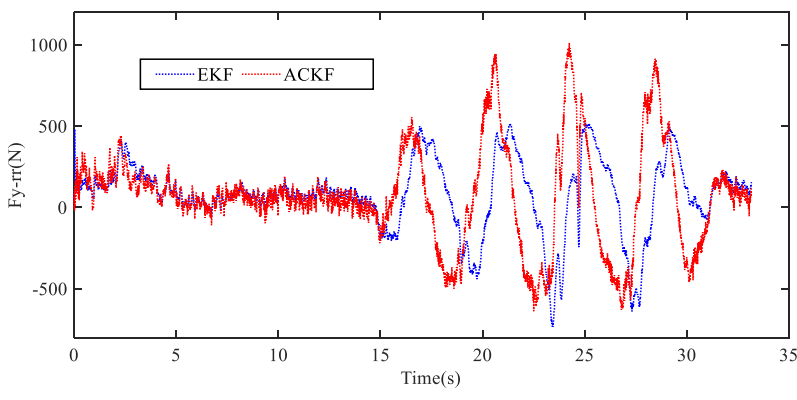

(d)

Figure 18 The lateral tire forces estimation in the simulation: (a) front left wheel, (b) front right wheel, (c) rear left wheel, (d) rear right wheel.

\section{Conclusions}

A novel observation algorithm to simultaneously estimate the body sideslip angles, yaw rate and lateral tire forces of all the four wheels is proposed in this paper. It mainly consists of four blocks: an observer block for body sideslip angle by ACKF, a correction block for body sideslip by APIO, a lateral tire force observer block and lateral tire force distribution block. Main contributions of this paper can be summarized as follows. Firstly, an ACKF is utilized to estimate the body sideslip angle and yaw rate. Secondly, the body sideslip angle is corrected by the measured and estimated yaw rate by APIO. Thirdly, the lateral tire force is estimated by ACKF and distributed to four wheels. Moreover, the simulation and car test are carried out to demonstrate the proposed method.

Results of both the simulation and real car test show that the proposed method performs well in both linear and nonlinear states.

\section{Declaration}

\section{Acknowledgements}

The authors sincerely thanks to Professor Z C He of Hunan University for his critical discussion and reading during manuscript preparation.

\section{Funding}

The project is supported by the National Natural Science Foundation of China (Grant No. U1864207), the Science Fund of State Key Laboratory of Advanced Design and Manufacturing for Vehicle Body (Grant No. 71975004), Changsha Science and technology Program (Grant No. kq1907104).

\section{Availability of data and materials}

The datasets supporting the conclusions of this article are included within the article.

\section{Authors' contributions}

The author' contributions are as follows: Zhen-Yu Zhang and Bao-lv Wei was in charge of the whole trial; Zhen-Yu Zhang wrote the manuscript; Zhi-Cheng HE and En-Lin Zhou assisted with sampling and laboratory analyses.

\section{Competing interests}

The authors declare no competing financial interests.

\section{Consent for publication}

Not applicable

\section{Ethics approval and consent to participate}

Not applicable

\section{References}

[1] B Y Zhang, C F Zong, G Y Chen, et al. A Novel Integrated Stability Control Based on Differential Braking and Active Steering for Four-axle Trucks. Chinese Journal of Mechanical Engineering, 2019, 32(12): 121. 
[2] Y Q Zhao, H Q Li, F Lin, et al. Estimation of Road Friction Coefficient [21] B L Boada, M J L Boada, V Diaz. Vehicle sideslip angle measurement in Different Road Conditions Based on Vehicle Braking Dynamics. Chinese Journal of Mechanical Engineering, 2017, 30(4): 982-990.

[3] S Cheng, L Li, B J Yan, et al. Simultaneous estimation of tire sideslip angle and lateral tire force for vehicle lateral stability control. Mechanical Systems and Signal Processing, 2019, 132: 168-182.

[4] S Cheng, L Li, B J Yan, et al. Simultaneous estimation of tire sideslip based on sensor data fusion using an integrated ANFIS and an Unscented Kalman Filter algorithm. Mechanical Systems and Signal Processing, 2016, 72-73: 832-845.

[22] X J Jin, G D Yin. Estimation of lateral tire-road forces and sideslip angle for electric vehicles using interacting multiple model filter approach. Journal of the Franklin Institute, 2015, 352(2): 686-707. angle and lateral tire force for vehicle lateral stability control. [ Mechanical Systems and Signal Processing, 2019, 132: 168-182.

23] I Arasaratnam, S Haykin. Cubature kalman filters. IEEE Transactions on Automatic Control, 2009, 54(6): 1254-1269.

[5] T Chen, X Xu, L Chen, et al. Estimation of longitudinal force, lateral [24] J Zarei, E Shokri. Convergence analysis of non-linear filtering based on vehicle speed and yaw rate for four-wheel independent driven electric vehicles. Mechanical Systems and Signal Processing, 2018, 101: 377388.

[6] T Chen, L Chen, Y F Cai, et al. Estimation of driving states based on pseudo-measurements of longitudinal force for distributed drive electric vehicles. Journal of Mechanical Engineering, 2019, 55(18): 86-94. (in Chinese)

[7] G Q Geng, B Y Wei, H B Jiang, et al. A research on driving state Estimation for distributed drive electric vehicle based on NA-EKF. Automotive Engineering, 2018, 40(7): 770-776. (in Chinese)

[8] Z Y Zhang, S Z Zhang, C X Huang, et al. State estimation of distributed drive electric vehicle based on adaptive extended Kalman filter. Journal of Mechanical Engineering, 2019, 55(6): 156-165. (in Chinese)

[9] G Reina, A Messina. Vehicle dynamics estimation via augmented Extended Kalman Filtering. Measurement, 2019, 133: 383-395.

[10] C Lin, G Wang, W K Cao, et al. A study on the vehicle state estimation for a distributed-drive EV based on LO-EKF algorithms. Automotive Engineering, 2014, 36(11): 1316-1320. (in Chinese)

[11] T Chen, L Chen, X Xu, et al. Estimation of longitudinal force and sideslip angle for intelligent four-wheel independent drive electric vehicles by observer iteration and information fusion. Sensors, 2018, 18(4).

[12] D W Pi, N Chen, J X Wang, et al. Design and evaluation of sideslip angle [31] observer for vehicle stability control. International Journal of Automotive Technology, 2011, 12(3): 391-399.

[13] L Li, J Gang, R Xu, et al. A variable structure extended Kalman filter for vehicle sideslip angle estimation on a low friction road. Vehicle System Dynamics: International Journal of Vehicle Mechanics and Mobility, 2014, 52(2): 280-308.

[14] L Li, J Gang, R Xu, et al. A variable structure adaptive extended Kalman filter for vehicle slip angle estimation. Int. J. Vehicle Design, 2011, 56: 161-185.

[15] A K Madhusudhanan, M Corno, E Holweg, et al. Vehicle sideslip estimator using load sensing bearings. Control Engineering Practice journal, 2016, 54: 46-57.

[16] W Liu, H W He, F C Sun, et al. Vehicle state estimation based on Minimum Model Error criterion combining with Extended Kalman Filter. Journal of the Franklin Institute, 2016, 353(4): 834-856.

[17] L Torres, G Besançon, D Georges. EKF-Like Observer With Stability for a Class of Nonlinear Systems. IEEE Transactions on Automatic Control, 2012, 57(6): 1570-1574.

[18] X D Liu, L Y Li, Z Li, et al. Stochastic Stability Condition for the Extended Kalman Filter With Intermittent Observations. IEEE Transactions on Circuits and Systems II: Express Briefs, 2017, 64(3): 334-338.

[19] W Q Zhou, X Qi. State estimation of vehicle based on multi-sensors information fusion. Automotive Engineering, 2019, 41(1): 7-13. (in Chinese).

[20] Z F Wang, Y C Qin, L Gu, et al. Vehicle system state estimation based on adaptive unscented Kalman filtering combing with road classification. IEEE Access, 2017, 5: 27786-27799. cubature Kalman filter. IET Science, Measurement and Technology, 2015, 9(3): 294-305.

[25] J Shi, G Q Qi, Y Y Li, et al. Stochastic convergence analysis of cubature Kalman filter with intermittent observations. Journal of Systems Engineering and Electronics, 2018, 29(4): 823-833.

[26] T R Wanasinghe, G K I Mann, R G Gosine. Stability analysis of the discrete-time cubature Kalman filter. Proceedings of the IEEE Conference on Decision and Control, 54rd IEEE(Cdc), 2015.

[27] J L Ding, J Xiao, T Zhao. Adaptive CKF strong tracking filter and application. Electric Machines and Control, 2015, 19(11): 111-120. (in Chinese)

[28] G Li, Y Wang, C F Zong. Driving state estimation of electric vehicle with four-wheel-hub-motors. Automotive Engineering, 2018, 40(2): 150-155. (in Chinese)

[29] J X Zhang, J Li. Vehicle state estimation based on interactive multiple model and cubature Kalman filter. Automotive Engineering, 2017, 39(9): 977-983. (in Chinese)

[30] Y Wang, G D Yin, K K Geng, et al. Tire lateral forces and sideslip angle estimation for distributed drive electric vehicle using noise adaptive cubature Kalman filter. Journal of Mechanical Engineering, 2019, 55(22): 103-1112. (in Chinese)

[31] T Chen, Y F Cai, L Chen, et al. Design of vehicle running states-fused estimation strategy using Kalman filters and tire force compensation method. IEEE Access, 2019, 7: 87273-87287.

[32] H B Pacejka and I J Besselink. Magic formula tyre model with transient Properties. Vehicle System Dynamics, 1997, 27: 234-249.

[33] K H Nam, F Hiroshi, H Yoichi. Advanced motion control of electric vehicles based on robust lateral tire force control via active front steering. IEEE/ASME Transactions on mechatronics, 2014, 19(1): 289-299.

\section{Biographical notes}

Zhi-Cheng He, born in 1983, is currently an associate professor at College of Mechanical and Vehicle Engineering, Hunan University, China. He received his $\mathrm{PhD}$ from Hunan University, China, in 2011. His research interests include man-machine system and intelligent robotics.

Tel: +0731-88821445; E-mail: hezhicheng815@gmail.com, hezhicheng815@163.com

Zhen-Yu Zhang, born in 1995, is currently a master at College of Mechanical and Vehicle Engineering, Hunan University, China. E-mail: zhangzhenyuhuda@163.com

En-Lin Zhou, born in 1985, is currently a PhD at College of Mechanical and Vehicle Engineering, Hunan University, China. E-mail:tenrey18@163.com

Bao-lv Wei, born in 1978, is currently a $\mathrm{PhD}$ at College of Mechanical and Vehicle Engineering, Hunan University, China.

E-mail: baolv.wei@sgmw.com.cn 
Figures

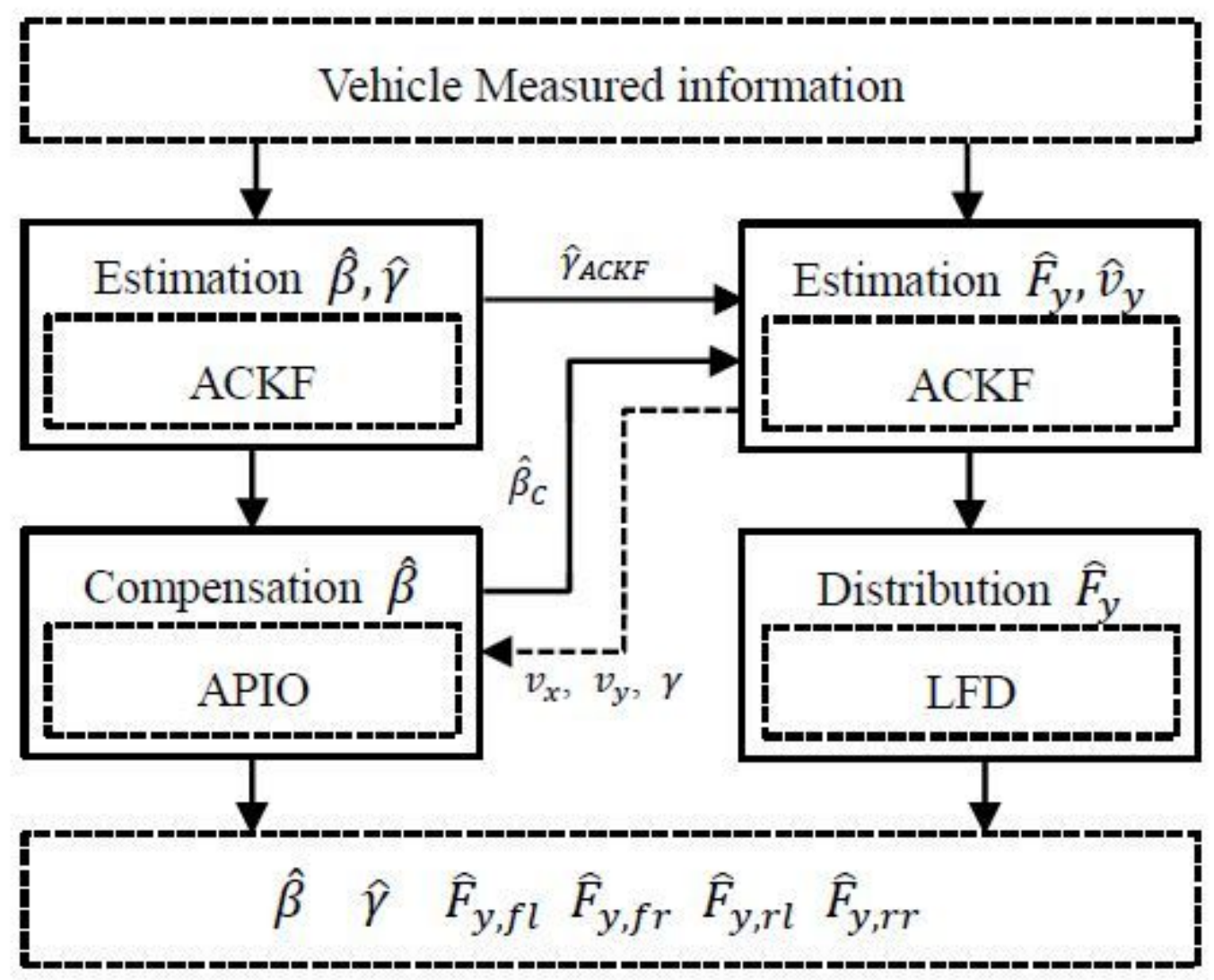

Figure 1

Architecture of the method for state estimation 


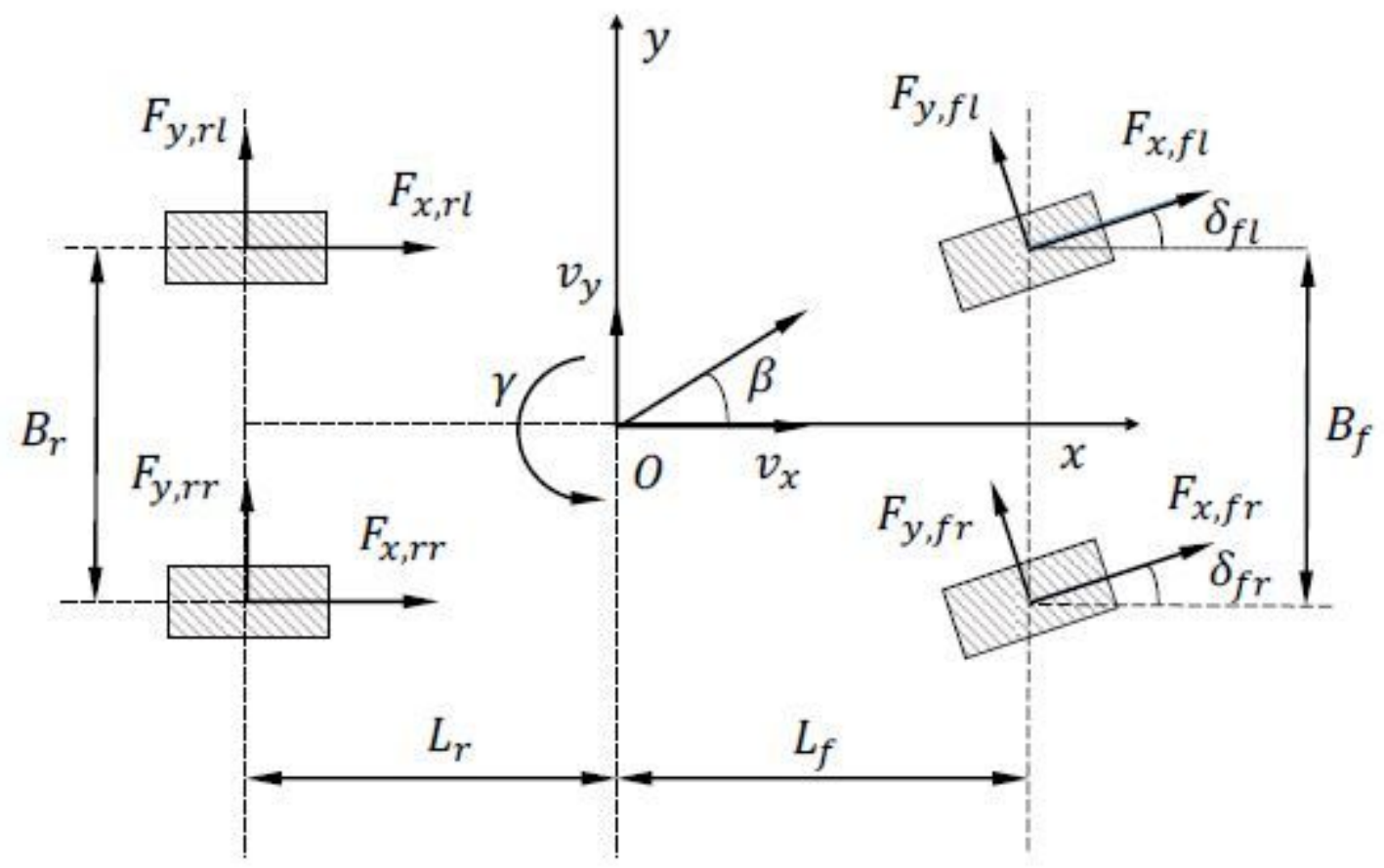

Figure 2

Vehicle dynamic model

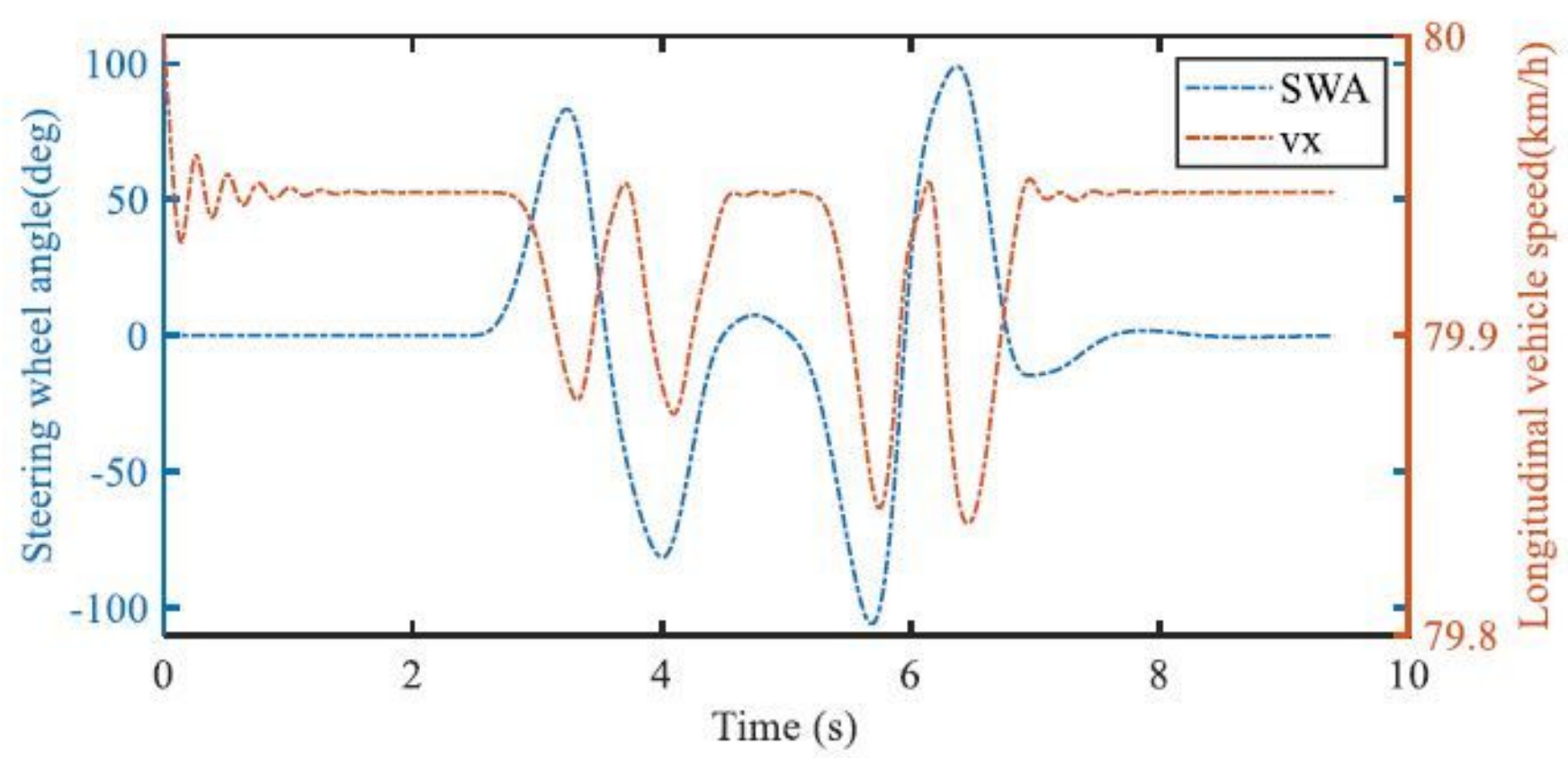

Figure 3

The steering wheel angle and longitudinal vehicle speed 


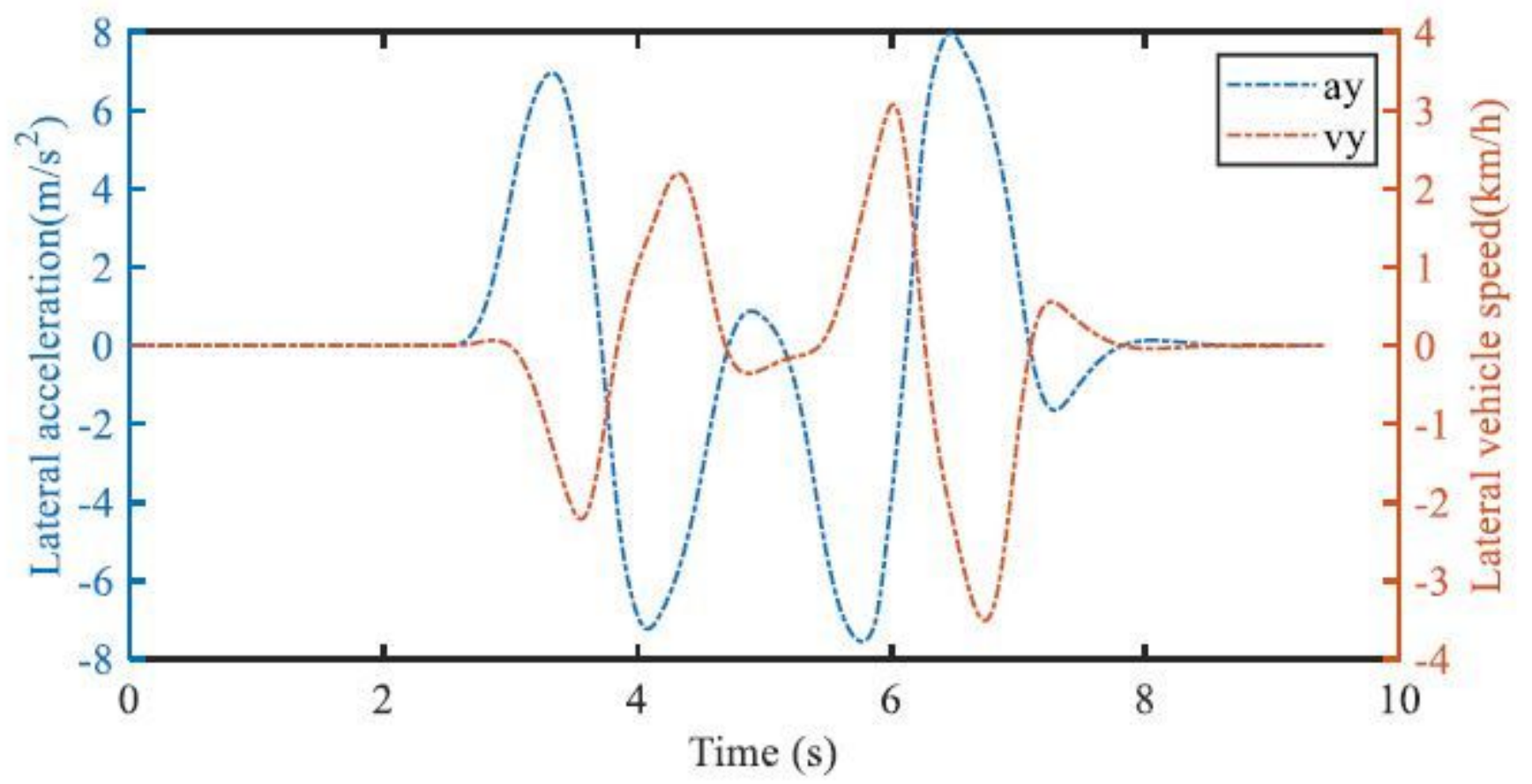

Figure 4

The lateral acceleration and lateral vehicle speed

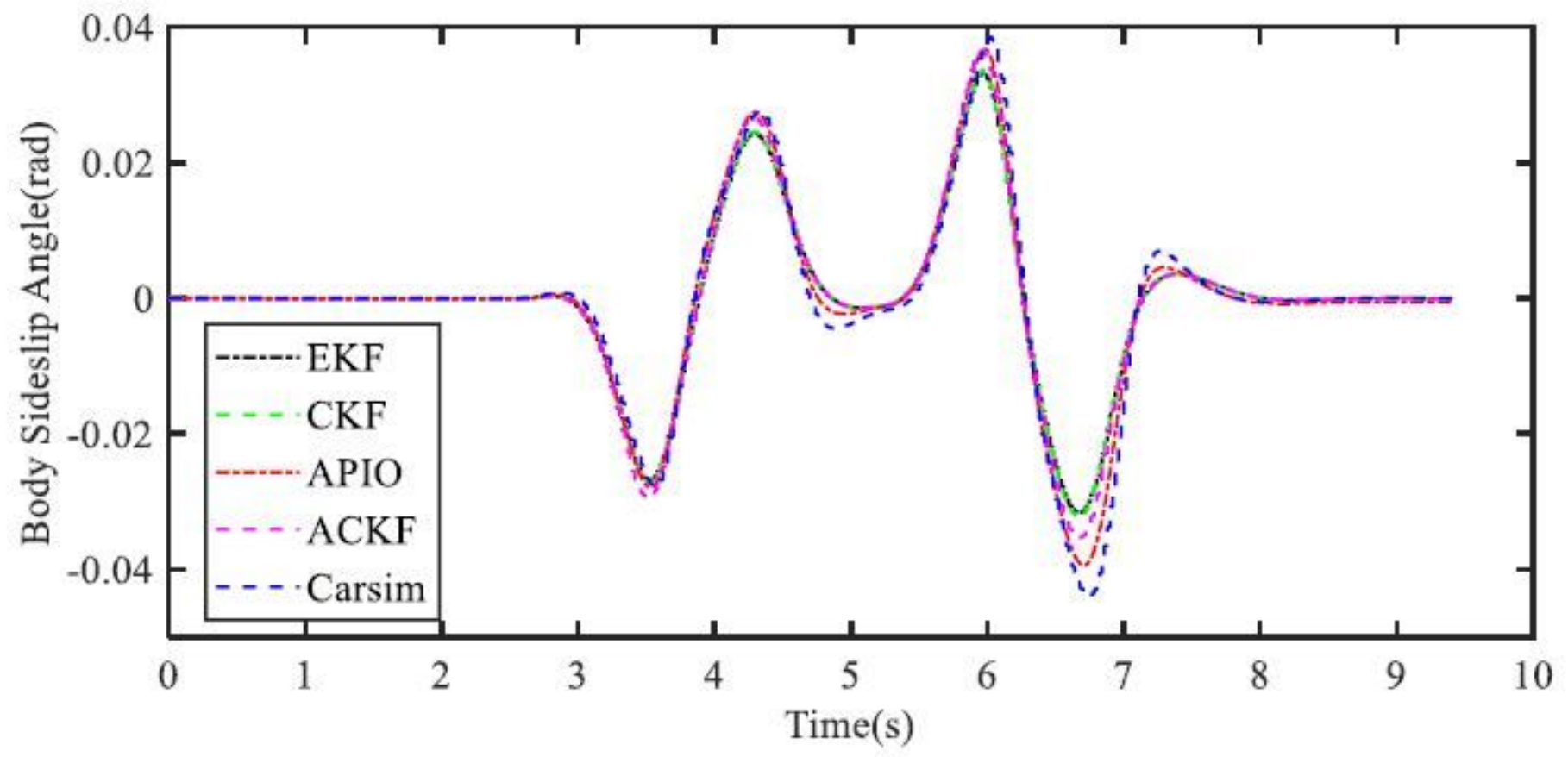

Figure 5

body sideslip angle estimated by ACKF and EKF 


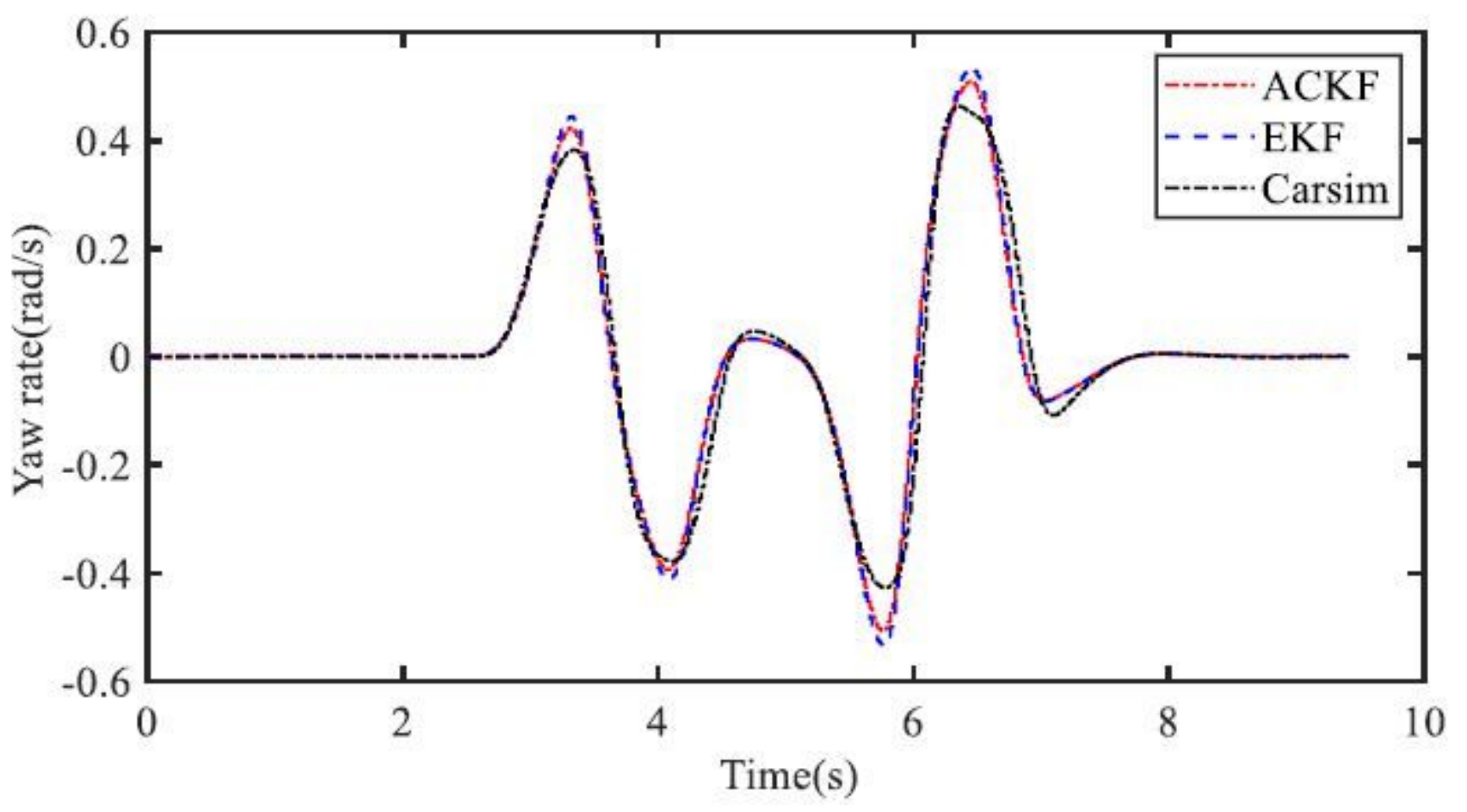

Figure 6

The yaw rate estimated by ACKF and EKF 


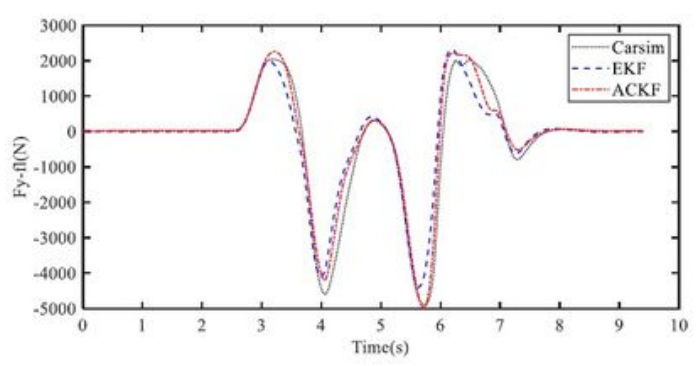

(a)

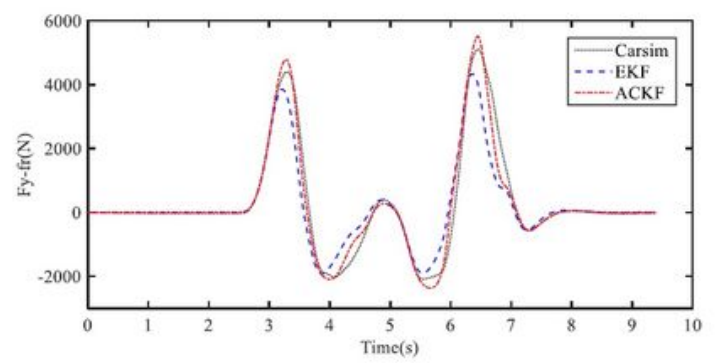

(b)

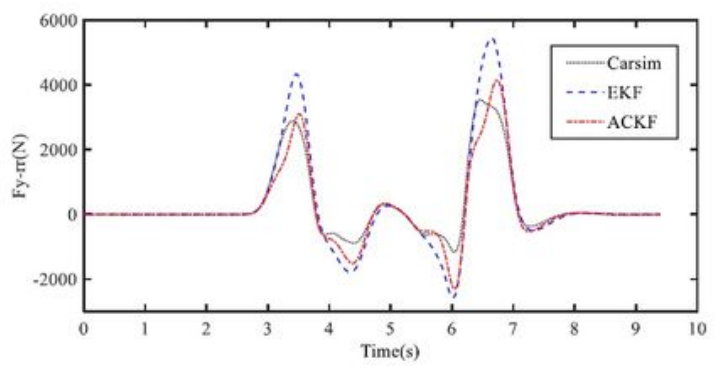

(c)

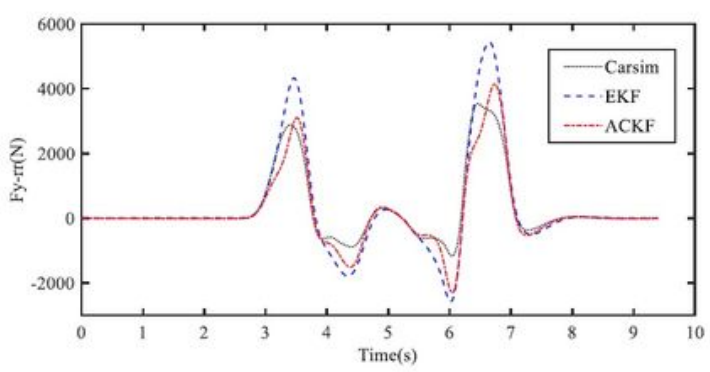

(d)

Figure 7

The lateral tire forces estimation in the simulation: (a) front left wheel, (b) front right wheel, (c) rear left wheel, (d) rear right wheel. 


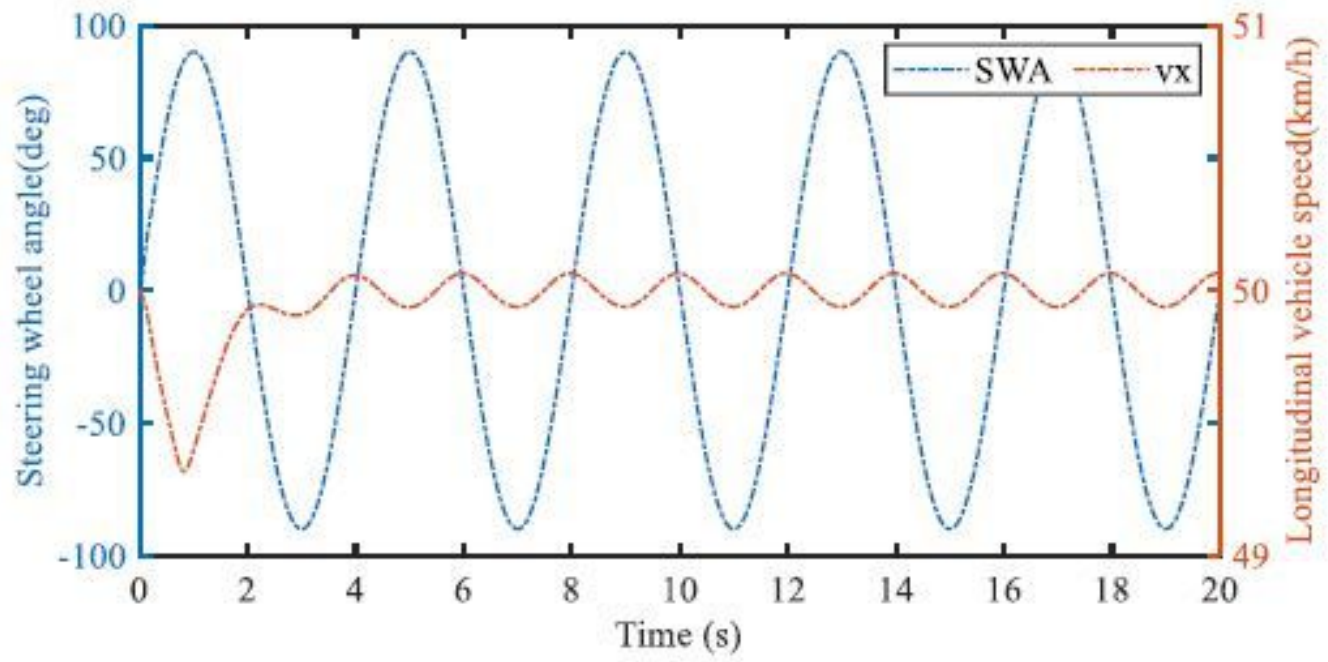

Figure 8

The steering wheel angle and longitudinal vehicle speed

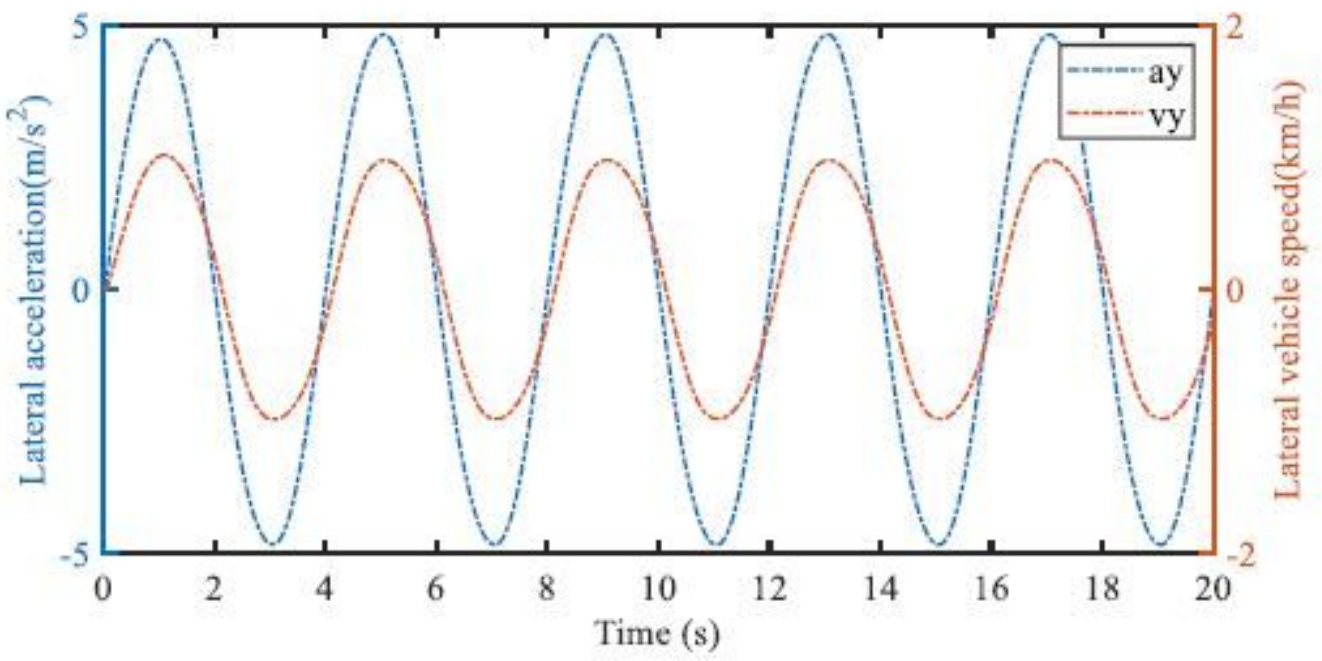

Figure 9

The lateral acceleration and lateral vehicle speed 


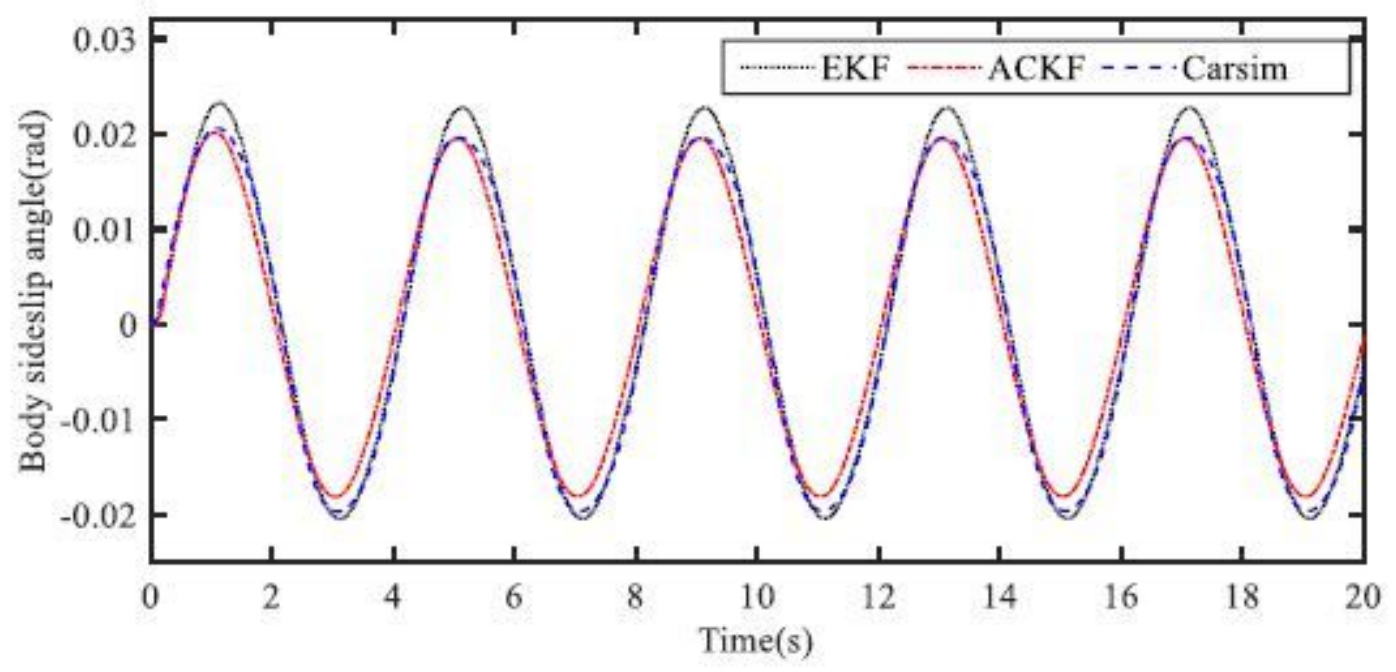

Figure 10

The body sideslip angle estimated by ACKF and EKF

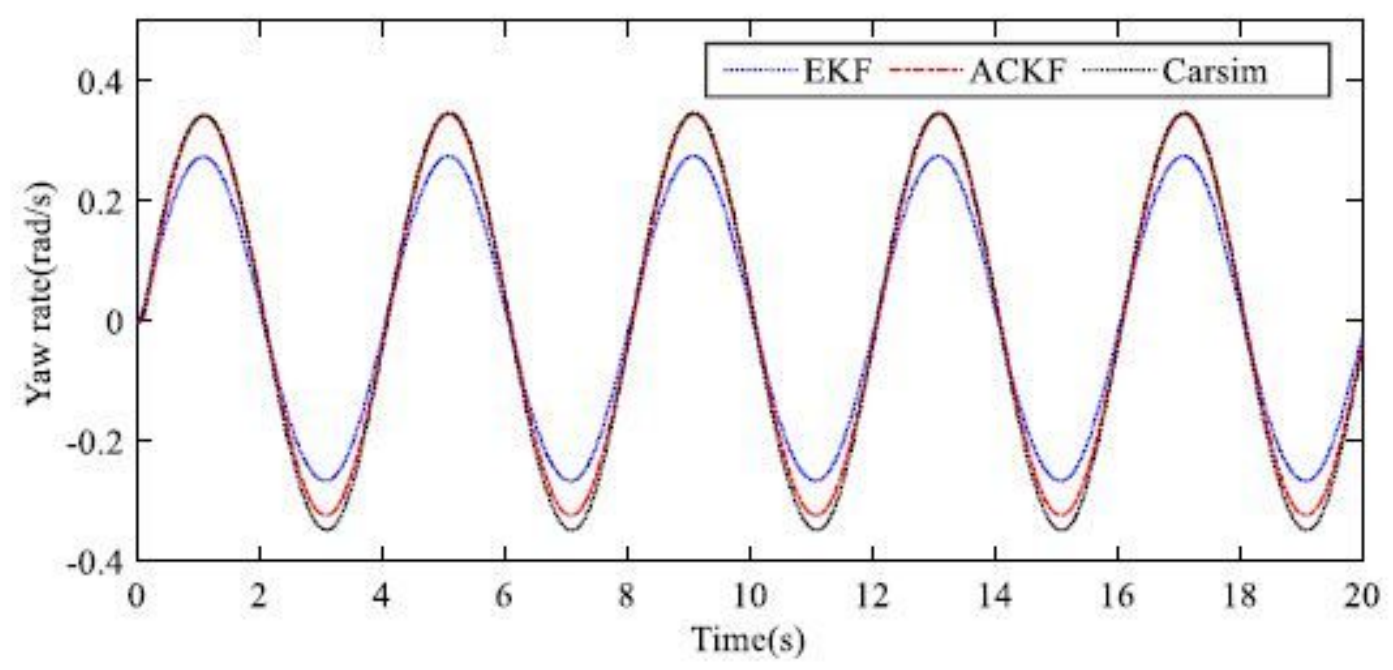

Figure 11

The yaw rate estimated by ACKF and EKF 


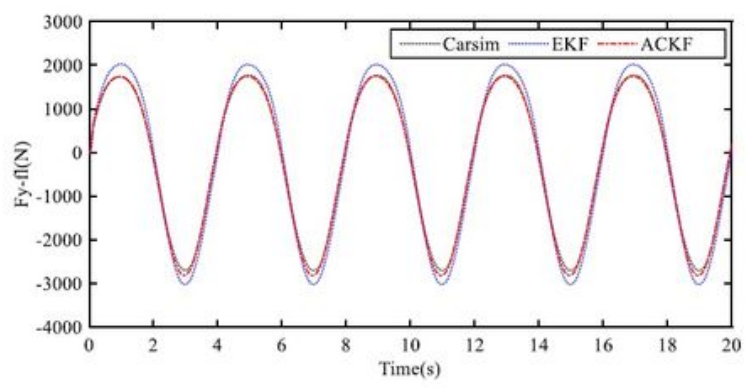

(a)

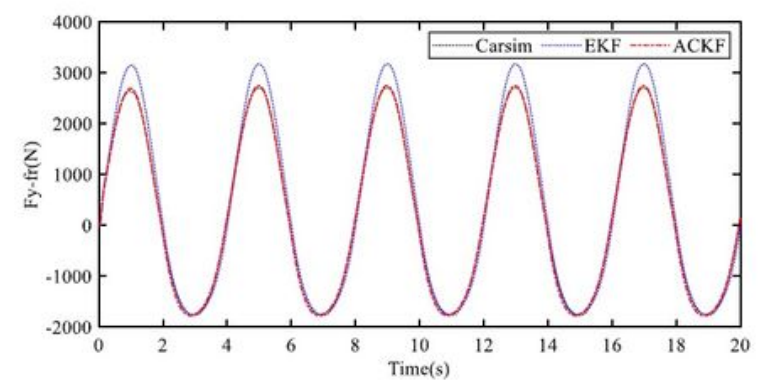

(b)

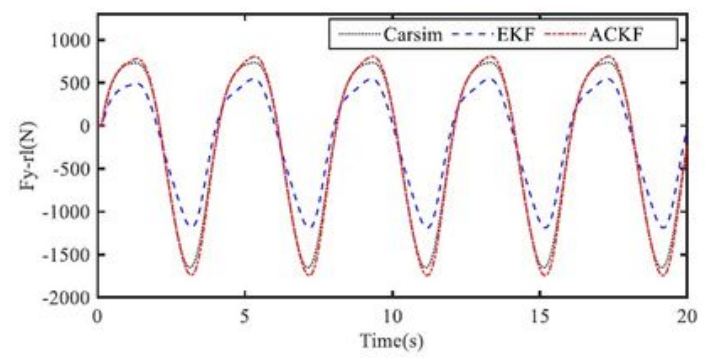

(c)

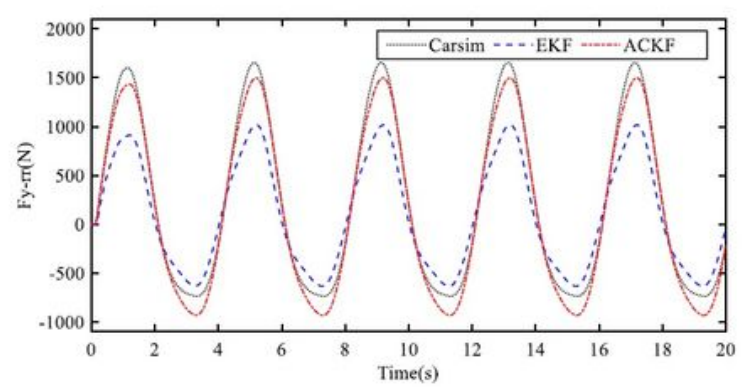

(d)

\section{Figure 12}

The lateral tire forces estimation in the simulation: (a) front left wheel, (b) front right wheel, (c) rear left wheel, (d) rear right wheel. 


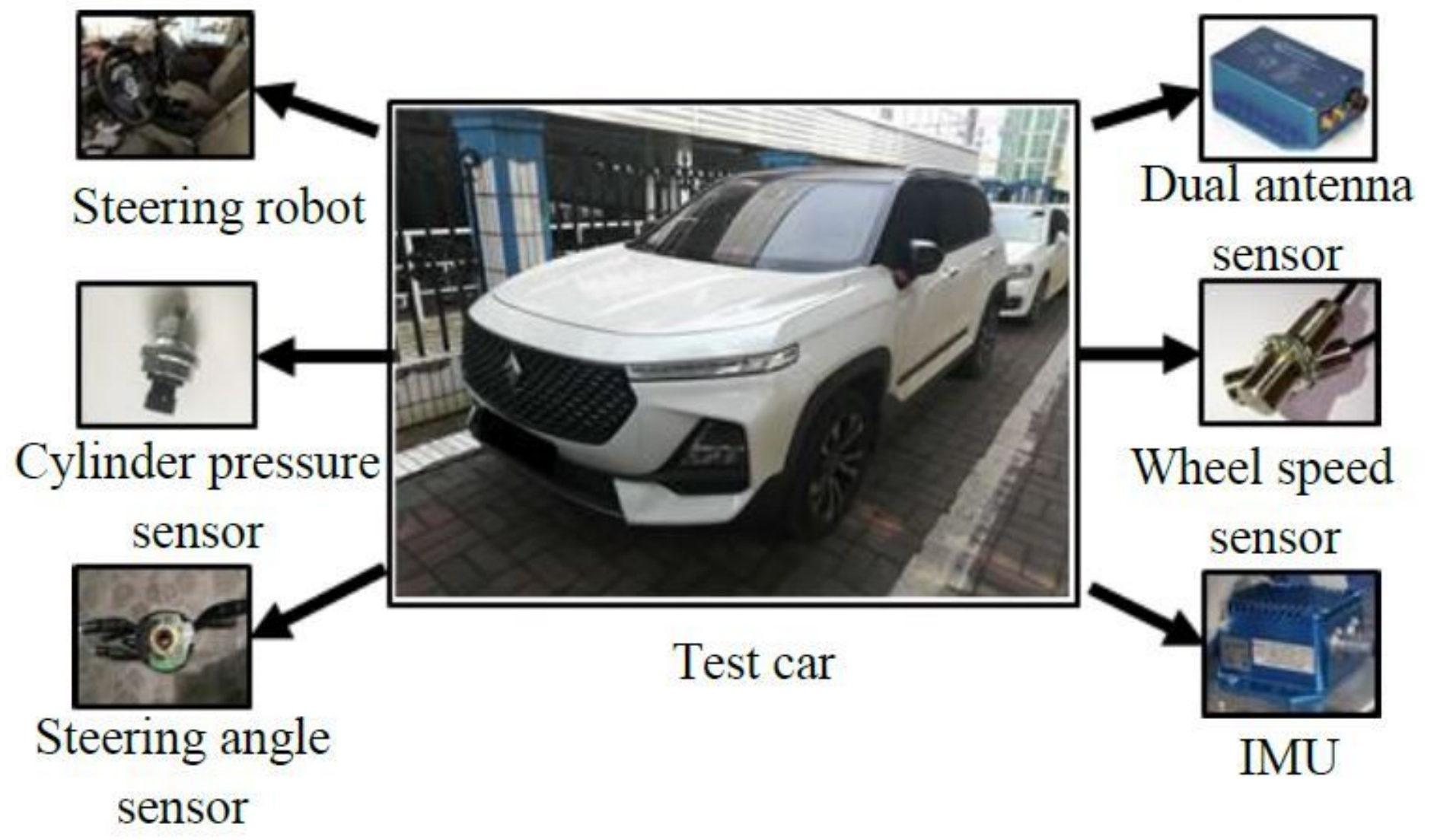

Figure 13

Test system

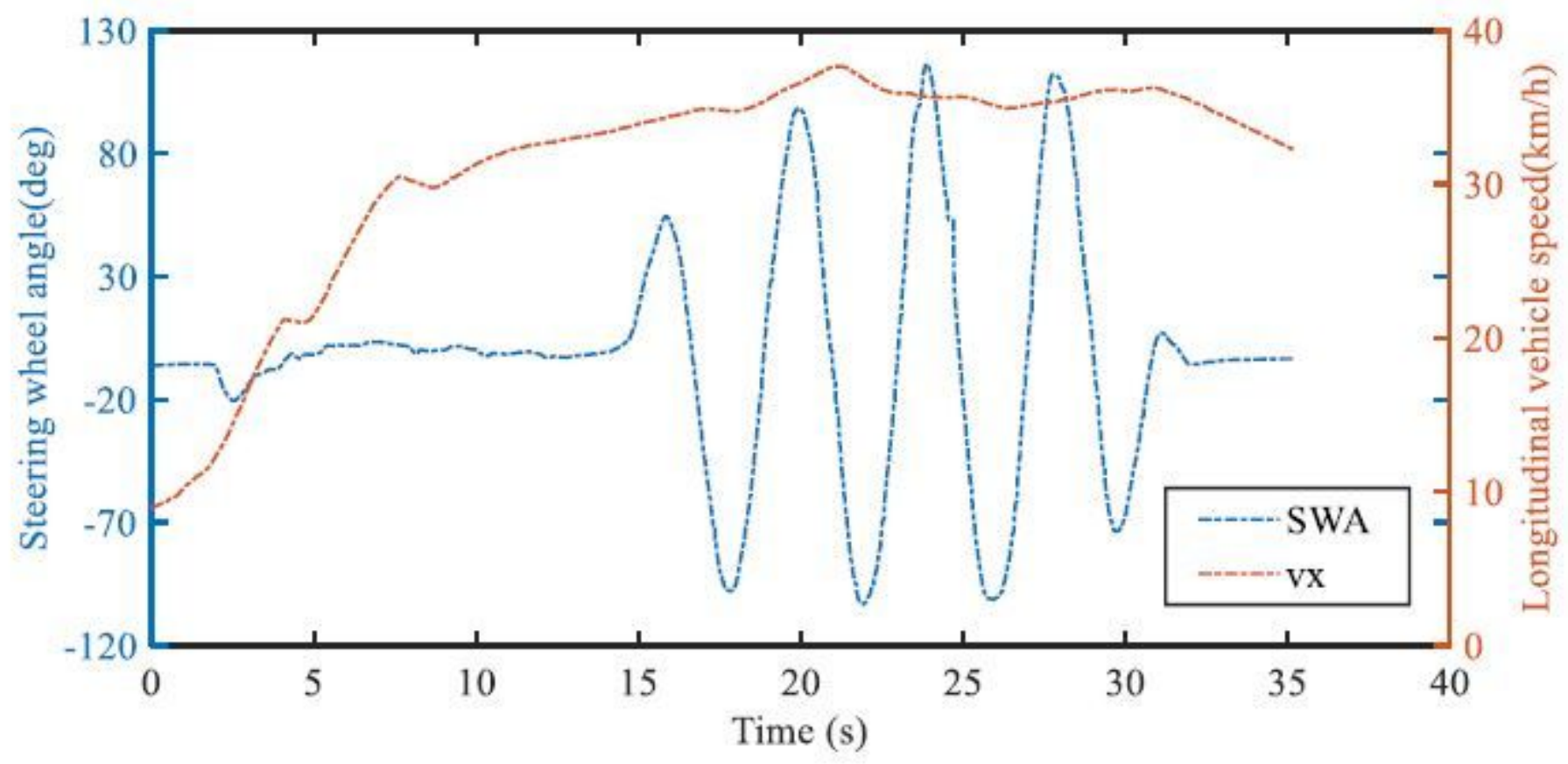


Figure 14

The steering wheel angle and longitudinal vehicle speed

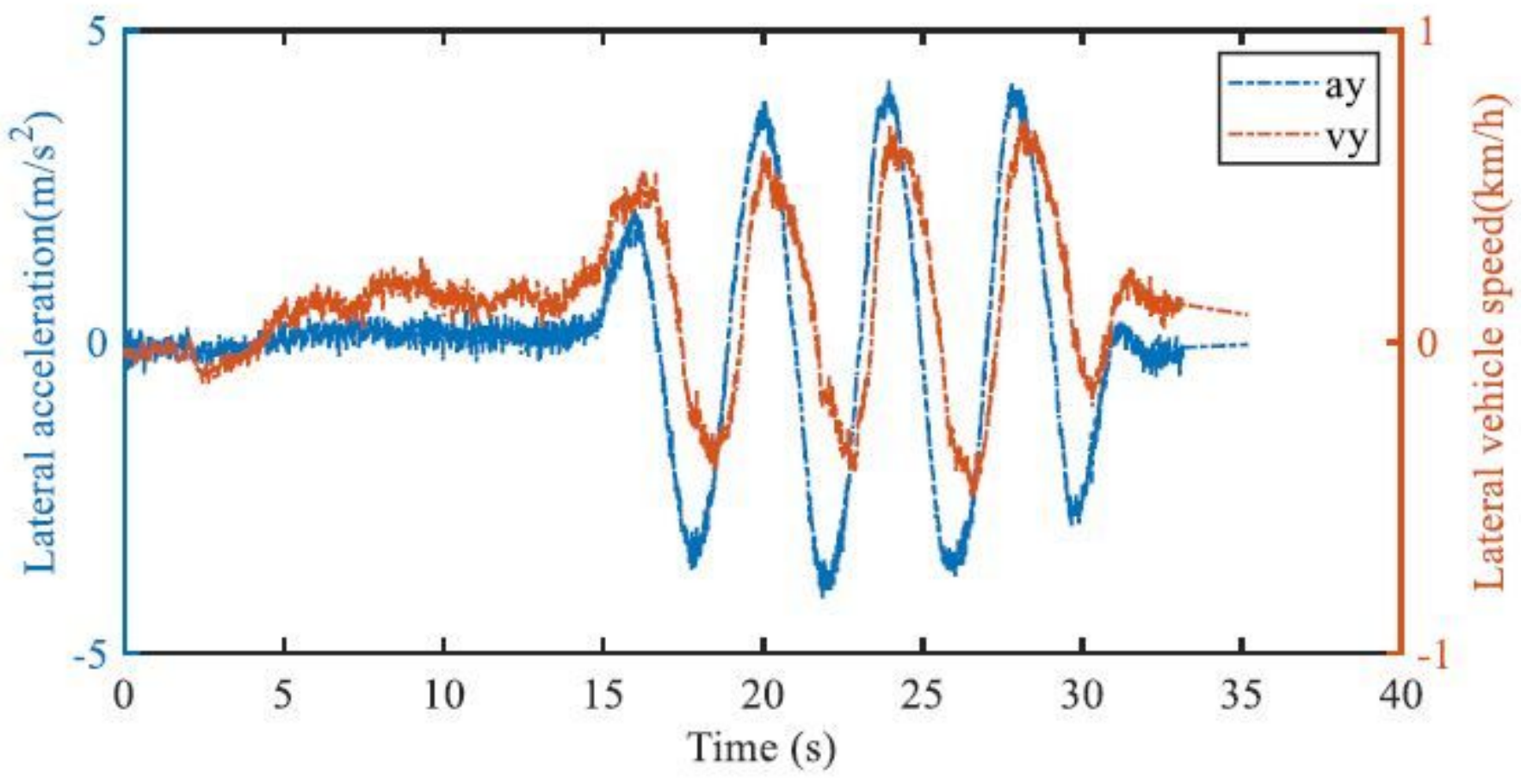

Figure 15

The lateral acceleration and lateral vehicle speed

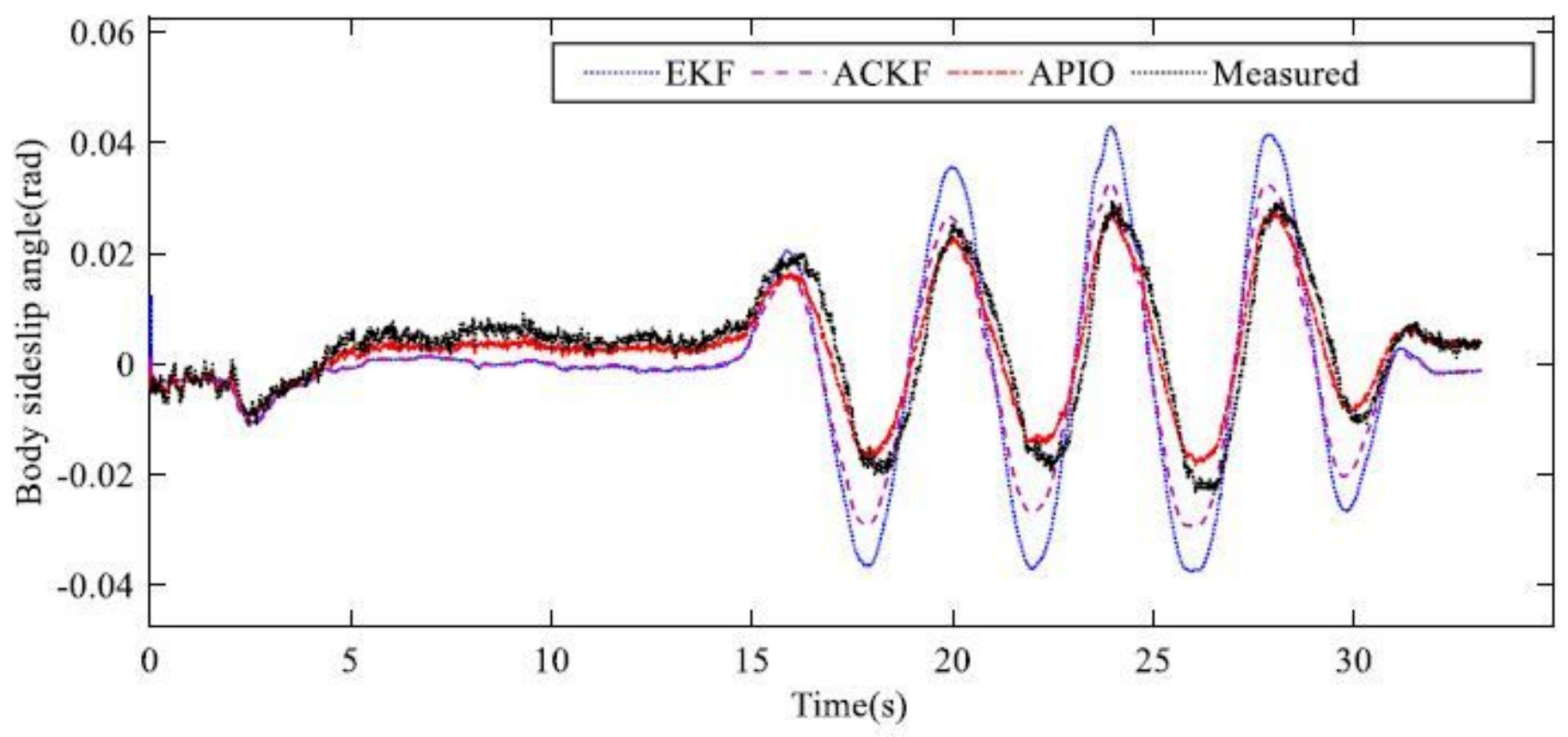

Figure 16 
The body sideslip angle estimated by ACKF and EKF

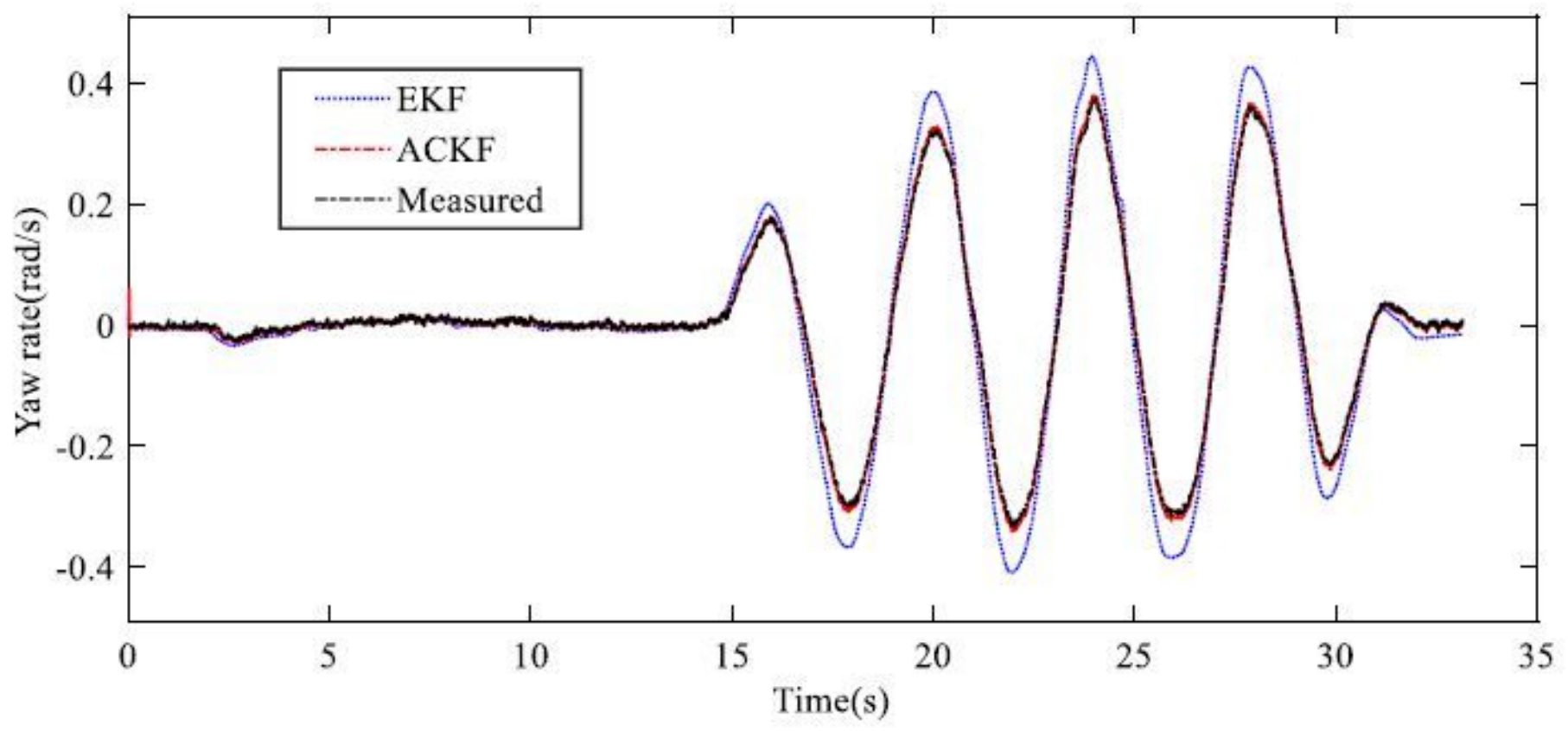

Figure 17

The yaw rate estimated by ACKF and EKF 


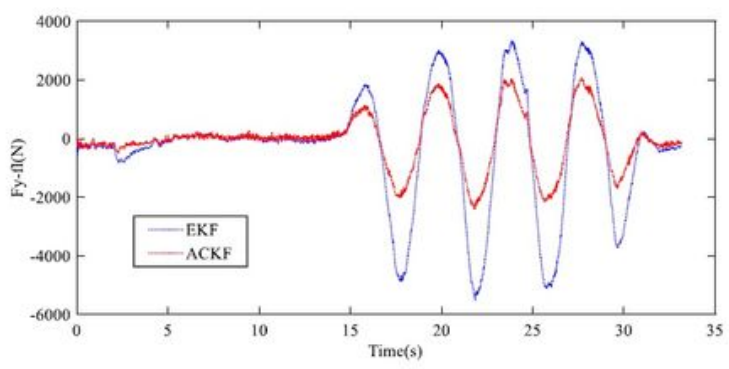

(a)

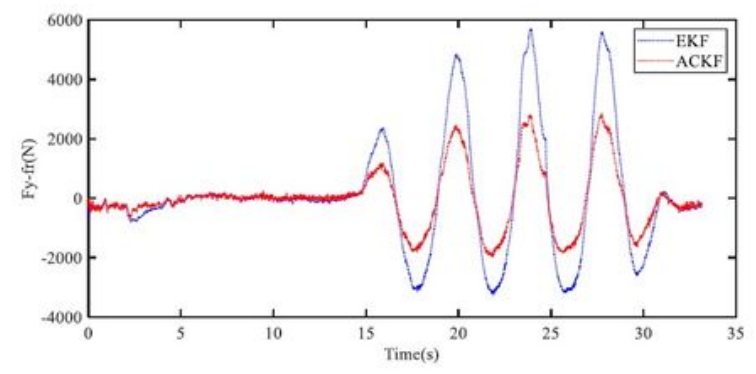

(b)

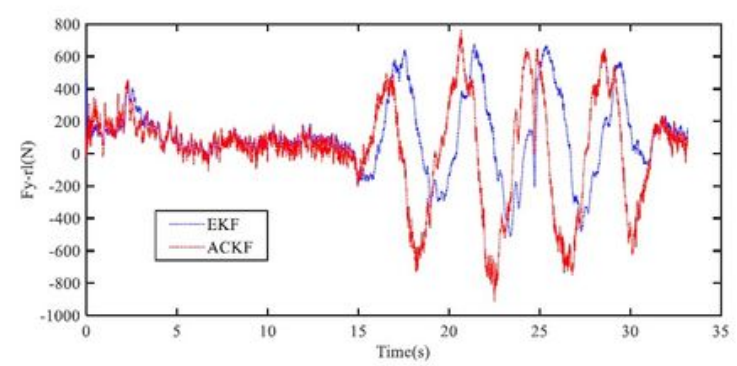

(c)

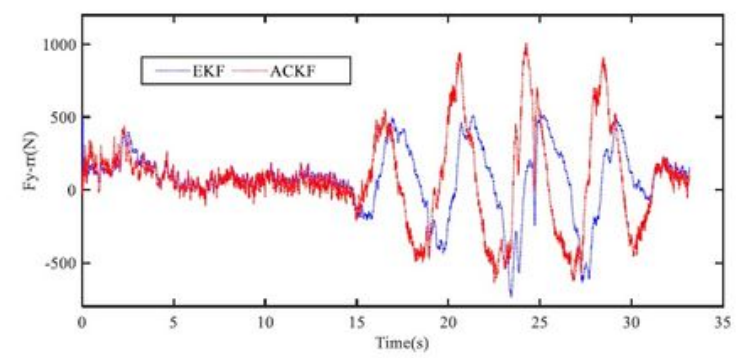

(d)

\section{Figure 18}

The lateral tire forces estimation in the simulation: (a) front left wheel, (b) front right wheel, (c) rear left wheel, (d) rear right wheel. 